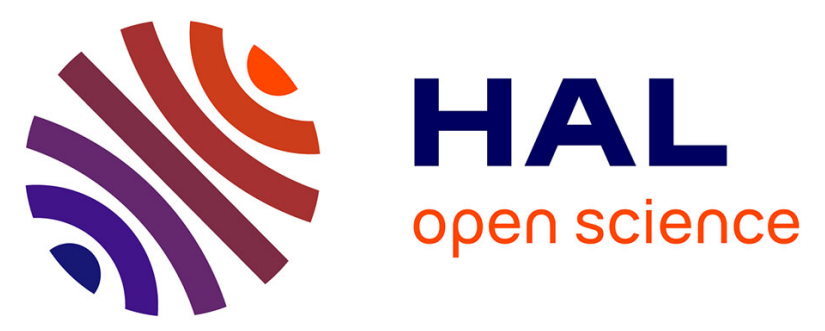

\title{
Filterability of exopolysaccharides solutions from the red microalga Porphyridium cruentum by tangential filtration on a polymeric membrane
}

\author{
Lisa Zaouk, Anthony Masse, Patrick Bourseau, Samir Taha, Murielle \\ Rabiller-Baudry, Sébastien Jubeau, Benoit Teychene, Jeremy Pruvost, Pascal \\ Jaouen
}

\section{To cite this version:}

Lisa Zaouk, Anthony Masse, Patrick Bourseau, Samir Taha, Murielle Rabiller-Baudry, et al.. Filterability of exopolysaccharides solutions from the red microalga Porphyridium cruentum by tangential filtration on a polymeric membrane. Environmental Technology, 2020, 41 (9), pp.1167-1184. 10.1080/09593330.2018.1523234 . hal-01880126

\section{HAL Id: hal-01880126 \\ https://hal-univ-rennes1.archives-ouvertes.fr/hal-01880126}

Submitted on 4 Oct 2018

HAL is a multi-disciplinary open access archive for the deposit and dissemination of scientific research documents, whether they are published or not. The documents may come from teaching and research institutions in France or abroad, or from public or private research centers.
L'archive ouverte pluridisciplinaire HAL, est destinée au dépôt et à la diffusion de documents scientifiques de niveau recherche, publiés ou non, émanant des établissements d'enseignement et de recherche français ou étrangers, des laboratoires publics ou privés. 
Publisher: Taylor \& Francis \& Informa UK Limited, trading as Taylor \& Francis Group Journal: Environmental Technology

DOI: $10.1080 / 09593330.2018 .1523234$

Filterability of exopolysaccharides solutions from the red microalga Porphyridium cruentum by tangential filtration on a polymeric membrane

ZAOUK Lisa ${ }^{(1,2)}$, MASSÉ Anthony ${ }^{(1)}$, BOURSEAU Patrick ${ }^{(1,}$, 3), TAHA Samir ${ }^{(2)}$, RABILLER-BAUDRY Murielle ${ }^{(4)}$, JUBEAU Sébastien ${ }^{(5)}$, Benoit TEYCHENÉ $^{(6)}$, PRUVOST Jérémy ${ }^{(1)}$, JAOUEN Pascal (1)

(1) GEPEA, Université de Nantes, CNRS, UMR 6144, Bd de l'Université, CRTT - BP 406, 44602 Saint-Nazaire Cedex, France

(2) AZM Center for Biotechnology Research and Its Applications, Laboratory of Applied Biotechnology, Lebanese University, EDST, Tripoli, Lebanon

(3) IRDL, Université de Bretagne Sud, CNRS, UMR 6027, Rue de Saint-Maudé, BP 92116, 56321 Lorient Cedex, France

(4) Univ Rennes, CNRS, ISCR (Institut des Sciences Chimiques de Rennes) - UMR 6226, F-35000 Rennes, France

(5) AlgoSource Technologies, 37 Bd de l'Université, 44600 Saint-Nazaire, France

(6) Université de Poitiers, CNRS, UMR 7285 (IC2MP), 4 rue Michel Brunet, TSA 51106, 86073 Poitiers Cedex 9, France

* Corresponding author. Email: patrick.bourseau@univ-ubs.fr 


\title{
Filterability of exopolysaccharides solutions from the red microalga Porphyridium cruentum by tangential filtration on a polymeric membrane
}

\begin{abstract}
The red microalga Porphyridium cruentum is exploited industrially for its exopolysaccharides (EPS) and pigments production. EPS produced by P. cruentum are partially released and dissolved into the surrounding environment, they can be recovered from the culture medium after removing the cells. This paper presents a parametric study of the ultrafiltration of EPS solutions on organic membrane. The EPS solutions were produced in conditions representative of an industrial production. They were filtered at lab-scale on a flat, PES $50 \mathrm{kDa}$ MWCO membrane in a complete recirculation mode of permeate and retentate. Permeate fluxtransmembrane pressure (TMP) curves were established up to the limiting flux for the filtration of solutions with various values of concentration in EPS ( 0.10 to $1.06 \mathrm{~kg}$ GlcEq. $\left.\mathrm{m}^{-3}\right)$, fluid tangential velocity $\left(0.3\right.$ to $1.2{\left.\mathrm{~m} . \mathrm{s}^{-1}\right)}^{2}$ and temperature $\left(20\right.$ and $\left.40{ }^{\circ} \mathrm{C}\right)$. The reversible and irreversible parts of fouling were evaluated for each experiment and the critical flux was determined for an intermediate EPS concentration $\left(0.16 \mathrm{~kg} \mathrm{GlcEq} \cdot \mathrm{m}^{-3}\right)$. The results showed that EPS solutions had a strong fouling capacity. When filtering the lowest concentrated solution $\left(0.10 \mathrm{~kg} \mathrm{GlcEq} \cdot \mathrm{m}^{-3}\right)$ with moderate fouling conditions, the overall fouling resistance was approximately half of the membrane and the share of irreversible/reversible fouling was 88 and $12 \%$. Howeyer, the part of reversible fouling becomes predominant when approaching the limiting flux. Permeate fluxes which were obtained allow to estimate that a VRR of approximately 10 could be obtained when concentrating EPS solutions using PES membranes in flat or tubular modules but not in spiral-wound.
\end{abstract}

Keywords: Porphyridium cruentum; exopolysaccharides (EPS); ultrafiltration; PES 50 kDa MWCO membrane; fouling. 
Word count : 9151 (main part only-abstract, acknowledgement, references, appendices, tables and figures excluded) 


\section{Introduction}

A wide range of microalgae and cyanobacteria is able to produce large amounts of extracellular polymeric substances, which are mainly composed of polysaccharides, proteins and/or other biopolymers in different proportions. This is the case for most of the red marine unicellular microalgae which are encapsulated within a sulfated polysaccharide gel that provides cells with protection against desiccation, predators and environmental extremes due to its stability to temperature, $\mathrm{pH}$ and salinity [1]. During the growth, the outer part of this gel undergoes dissolution into the culture medium (soluble fraction), 50 to $70 \%$ of the polysaccharide remains attached to the cell (bound fraction) [2]. The red microalgae belonging to the Porphyridium genus especially P. cruentum are currently cultivated on a large scale for the production of exopolysaccharides and pigments [3] and may have a future for lipid-based industrial applications due to their original and/or highly specific lipid profiles [2]. The amount of exopolysaccharides (EPS) produced and the distribution between bound and dissolved fractions change according to the culture conditions, physiological state of microalgae, cultivation mode, light quality and intensity, salinity, etc. $[2,4]$. The production of EPS is particularly enhanced during the stationary phase of growth [5], soluble polysaccharide concentrations ranging from 0.10 to $0.70 \mathrm{~kg} . \mathrm{m}^{-3}$ have been reported in the literature [6-9]. Higher values up to 4.6 kg. $\mathrm{m}^{-3}$ have also been reported [10] but the status of the EPS is not clear and could include not only the soluble part of EPS but also some or all of the cell-bound part.

The apparent molecular mass of Porphyridium sp. EPS has been estimated by SEC-HPLC to $2-7 \times 10^{6}$ Da [1] but the results might be influenced by the tendency of molecules to aggregate in solutions [11]. These EPS have specific structures with in particular an acidic character due to the presence of glucuronate, sulfate and carboxylic groups [12] which give them biological activities which are of high interest and value to the cosmetic, nutraceutical and therapeutic industries. Natural polysaccharides are currently gaining interest for recent applications such as novel food, pharmaceutical and cosmetic industries. As the growing demand cannot be fully satisfied by conventional available sources such as red and brown macroalgae, EPS from Porphyridium species that have similar techno-functional properties, they could penetrate the large market of phycocolloids provided that the technologies for both their autotrophic solar production 
at high cell densities, and their downstream processing attain the breakeven point $[1,4]$. The industrial exploitation of $P$. cruentum EPS is in particular limited because of their low concentration in rich salt culture media [13]. However, for large-scale culture, EPS recovery should be part of a strategy of a complete bio-refinery of the microalga culture in order to mitigate the production cost.

The conventional extraction processes of microalgae EPS from their culture medium consists in a cells removing step by centrifugation or microfiltration followed by an alcoholic precipitation (see the review on microalgae EPS by Delattre et al. [4]). However, the authors pointed out that the development of membrane processes is a good alternative for the desalting and concentration of EPS solutions before their drying even if they constitute a very limited step because of their high viscosity and accordingly very high cost. They indicated also that alcoholic precipitation is not really appropriate to purify EPS from marine microalgae as they will often be contaminated by salts which coprecipitate with them.

Ultrafiltration (UF) has been poorly investigated for the extraction and the purification of EPS from culture supernatants of microalgae. Li et al. [14] isolated EPS from the spent culture media of 8 photosynthetic microorganisms (cyanobacteria, green algae and a diatom), with the aim to test their bioactive (antitumor) properties. The cellfree media clarified by a dead-end microfiltration were filtered by tangential UF at a pilot-scale on an organic membrane in polyethersulfone (PES) with a $5 \mathrm{kDa}$ molecular weight cut-off (MWCO). A high EPS isolation efficiency was achieved with volume reduction ratio (VRR) of 20 or 40. Although the initial EPS concentration was not specified, the work demonstrated the feasibility of concentratingf microalgal EPS from clarified cell-free culture media. More recently, Patel et al. [13] found that a tangential flow filtration system used in diafiltration mode was a potential technology to collect and desalt selectively the EPS of $P$. cruentum from cell-free culture media. On the other hand, Marcati et al. [3] separated B-phycoerythrin and soluble polysaccharides from $P$. cruentum biomass with a high polysaccharidic content $\left(0.63 \mathrm{~kg} \cdot \mathrm{m}^{-3}\right)$ by a two-step membrane process using PES flat membranes. In addition, some authors have highlighted the role of EPS in fouling during the harvesting of the microalga P. purpureum [15] or the cyanobacterium A. platensis [16]. Indeed, even at low concentrations, microalgae 
EPS form highly viscous solutions that can exhibit high stickiness and propensity to form slimy gels which could severely foul the membranes.

Membrane fouling is often a critical point of the economic profitability of membrane processes because severe fouling penalizes both CAPEX and OPEX. Therefore, a good understanding of fouling is needed to adapt the filtration conditions in order to minimize fouling and filtrations costs. The critical flux concept was developed in that sense simultaneously by Field et al. [17] and Bacchin et al. [18]. These authors published 10 years later a joint paper in which they clarified the concept and distinguished 3 forms of critical flux [19]. The strong form $J_{c s}$ discriminates no fouling conditions from fouling conditions of any type, supposing that osmotic pressure effects and adsorption resistance are negligible. In practice, $\mathrm{J}_{\mathrm{cs}}$ is defined as the point at which the flux starts to deviate from the pure water flux-transmembrane pressure (TMP) line. The weak form $J_{c w}$ is observed in systems where an initial adsorption takes place independently of pressure and solvent transfer through the membrane and occurs immediately at the start of the operation, leading to a linear portion of the flux-TMP curve with a lower slope than the pure water flux line. Finally, the critical flux for irreversibility $J_{c i}$ discriminates fouling with respect to its irreversibility. Below it, only a concentration polarization layer exists, with an additional monolayer of adsorbed species in some cases. For colloidal systems, $J_{\mathrm{ci}}$ is linked to a physical phase transition from a dispersed phase (concentration polarization) to a condensed phase (multi-layer deposit).

This paper aims to estimate the filterability of EPS solutions issued from $P$. cruentum cultures with modules equipped with organic (PES) membranes known known to have lower investment costs and pumping rates than mineral membranes [Cheryan, chap. 5]. Firstly, we evaluated the influence of the main operating parameters (tangential velocity, transmembrane pressure, EPS concentration and temperature) on both permeate flux and membrane fouling at a lab-scale using a flat polymeric, PES $50 \mathrm{kDa}$ MWCO membrane. The extent and the type of fouling were then characterized in terms of fouling resistances and critical flux for irreversibility. Finally, the feasibility of the isolation of $P$. cruentum EPS by ultrafiltration on organic membranes at an industrial level will be discussed. 


\section{Experimental}

\subsection{Water and preparation of EPS solutions}

Water used for solutions preparation, membrane filtration and set-up flushing and cleaning is a type 2 (resistivity of $15 \mathrm{M} \Omega . \mathrm{cm}$ at $25^{\circ} \mathrm{C}$ ), pure water delivered by an Elix ${ }^{\circledR}$ Advantage System (Millipore). It's referred to as deionized water in the subsequent text.

The microalga $P$. cruentum (UTEX 161) was produced and harvested on the Algosolis microalgae R\&D facility (Saint-Nazaire, France, http://algosolis.com/). This facility is dedicated to the development of breakthrough technologies for mieroalgae culture and biorefinery and offers the infrastructure for investigations in conditions representative of industrial applications. P. cruentum was grown in a modified Bold's basal medium within a raceway $\left(1000 \mathrm{~L}, 10 \mathrm{~m}^{2}\right)$ operated in a batch mode. The fresh medium contained $15 \mathrm{~kg} \cdot \mathrm{m}^{-3}$ of $\mathrm{NaCl}$ and $3.4 \mathrm{~kg} \cdot \mathrm{m}^{-3}$ of other nutrients (see Table 1). The culture was maintained outdoor under solar flux at a $\mathrm{pH}$ of 7.5 and a temperature varying between 17 and $28{ }^{\circ} \mathrm{C}$ for culture $\mathrm{A}$, and between 20 and $25{ }^{\circ} \mathrm{C}$ for culture $\mathrm{B}$. The cultures were harvested when they were 22 and 18 days old respectively, the cells being in the stationary phase of growth. The biomass and the culture medium containing EPS were separated by centrifugation at $3000 \mathrm{~g}$ in a dynamic settler based on the Evodos spiral plate technology (Eyodos 10). The separation produced supernatants $\mathrm{A}$ and $\mathrm{B}$, respectively for cultures $A$ and B. A part of supernatant B was concentrated using a UF tubular ceramic membrane with a $50 \mathrm{~nm}$ mean pore diameter (Membralox P1940), the concentrate being referred as supernatant Bc. All the supernatants (A, B and Bc) were stored at $-20^{\circ} \mathrm{C}$.

Supernatants were thawed at $4{ }^{\circ} \mathrm{C}$ for 24 to 48 hours just before the preparation of the EPS solutions to be filtered. Characteristics of the four EPS solutions used in the work are summarized in Table 2. The EPS solution $\mathrm{S}_{\mathrm{A}}$ was obtained after a second centrifugation of supernatant A (Thermo Scientific Sorvall RC6 Plus, 2 times at $6000 \mathrm{~g}$, $15 \mathrm{~min}, 4^{\circ} \mathrm{C}$ ) in order to remove residual cell debris and had an EPS concentration of $0.10 \mathrm{~kg}$ GlcEq.m ${ }^{-3}$. The EPS solution $\mathrm{S}_{\mathrm{B}}$ was the raw supernatant $\mathrm{B}$ itself $(0.16 \mathrm{~kg}$ GlcEq. $\mathrm{m}^{-3}$ ). By the end, supernatant Bc underwent a second centrifugation (same conditions as for $\mathrm{S}_{\mathrm{A}}$ ) in order to remove precipitates that appeared after thawing, before 
being diluted with fresh modified BBM medium to obtain the solutions $\mathrm{S}_{\mathrm{Bcd} 1}(0.64 \mathrm{~kg}$ GlcEq. $\left.\mathrm{m}^{-3}\right)$ and $\mathrm{S}_{\mathrm{Bcd} 2}\left(1.06 \mathrm{~kg}\right.$ GlcEq. $\left.\mathrm{m}^{-3}\right)$.

Turbidity (O.D. at $750 \mathrm{~nm}$ ), dry matters DM, pH and conductivity of all solutions are closed. The main difference is the protein to EPS ratio which is approximately 2 to 3 times higher for $\mathrm{S}_{\mathrm{A}}$ and $\mathrm{S}_{\mathrm{B}}$ than for the concentrated solutions $\mathrm{S}_{\mathrm{Bcd} 1}$ and $\mathrm{S}_{\mathrm{Bcd} 2 \text {. }}$

\section{Insert Table 1 here}

\section{Insert Table 2 here}

\subsection{Assays}

Total sugar content was evaluated by a colorimetric method (spectrophotometer Jasco V630) after adding phenol and sulfuric acid as described by Dubois et al. [20]. The absorbance was read at $483 \mathrm{~nm}$ and the results were expressed in $\mathrm{kg} . \mathrm{m}^{-3}$ of D-glucose equivalent (kg GlcEq. $\mathrm{m}^{-3}$ ). Permeate samples were diafiltered using a $10 \mathrm{kDa}$ MWCO PES membrane (Biomax) at 2 bar in order to remove salts (mainly nitrates) that can adulterate the measuring results.

Protein content was quantified by the bicinchoninic acid (BCA) method using bovine serum albumin (Sigma-Aldrich, BCA1-1KT) as a standard. The absorbance was measured at $562 \mathrm{~nm}$. Cell-free medium were dried at $105^{\circ} \mathrm{C}$ and weighted until constant mass in order to obtain the dry weight. Total sugar and protein concentrations as well as the dry weight were all measured in triplicates.

Solutions turbidity was evaluated through the measurement of the optical density at $750 \mathrm{~nm}$. Their conductivity and $\mathrm{pH}$ were measured using a portable conductivity meter (Mettler Toledo ${ }^{T M}$ FG3 FiveGo ${ }^{\text {TM }}$ equipped with a probe LE703 and a pH portable meter (Mettler Toledo ${ }^{\mathrm{TM}}$ FG2 FiveGo $^{\mathrm{TM}}$ ), respectively.

Concentrations of salts in EPS solutions were measured by ionic chromatography. Prior to injection, EPS solutions underwent specific dilutions with deionized water then the diluted samples were filtered through $0.2 \mu \mathrm{m}$ cellulose acetate (Minisart ${ }^{\circledR} \mathrm{NML}$ syringe filter) in order to remove particulates. The anionic chromatograph Dionex - ICS 900 (Thermo Scientific) was equipped with a guard column AG9-HC $(4 \times 50 \mathrm{~mm})$ and a 
separation column AS9-HC $(4 \times 250 \mathrm{~mm})$. The eluent was a mixture of solution of $7.7 \mathrm{x}$ $10^{-3}$ mol.L $\mathrm{L}^{-1} \mathrm{Na}_{2} \mathrm{CO}_{3}$ and $1.3 \times 10^{-3} \mathrm{~mol} . \mathrm{L}^{-1} \mathrm{NaHCO}_{3}$, with a flow rate of $1.0 \mathrm{~mL} \cdot \mathrm{min}^{-1}$. The cationic chromatograph Dionex - ICS1100 (Thermo Scientific), had a guard column CG16 $(5 \times 50 \mathrm{~mm})$ and a separation column CS16 $(5 \times 250 \mathrm{~mm})$. The elution was performed with $17 \times 10^{-3}$ mol.L $\mathrm{L}^{-1} \mathrm{H}_{2} \mathrm{SO}_{4}$ at a flow rate of $1.2 \mathrm{~mL} \cdot \mathrm{min}^{-1}$. In order to regulate the stationary phase, a chemical suppressor AMMS $^{\circledR} 300,4 \mathrm{~mm}\left(25 \times 10^{-3}\right.$ mol.L $\mathrm{L}^{-1} \mathrm{H}_{2} \mathrm{SO}_{4}$ ) was used for the anion column, whereas the cationic column was equipped with an electrochemical suppressor $\operatorname{CSRS}^{\circledR} 300,4 \mathrm{~mm}$ (self-regeneration by electrolysis). Data acquisition and processing were accomplished with the Chromeleon ${ }^{\circledR} 7$ software. The detection signal was the conductivity of the eluted solution and the concentration of an ion was determined knowing the proportionality between the ion concentration and the areas under its chromatographic peak.

\subsection{Ultrafiltration runs}

\subsubsection{Membrane and ultrafiltration set-up}

A PES membrane with a $50 \mathrm{kDa}$ MWCO (Synder membranes, Vacaville, CA, USA, model MQ) was used in the study. The membrane was delivered as a roll from which 3 rectangular samples were cut for filtration processes. The initial water permeabilities and membrane resistances of samples are given in Table 3. To prevent drying of the membrane and entrapment of air bubbles in the membrane pores, samples were kept in the pilot filled with deionized water during the experiments campaign and water was renewed regularly in order to avoid bacterial contamination.

The experimental study was performed in a cross-flow lab-scale membrane unit (see Figure 1). The plate and frame module Rayflow 100 (Rhodia-Orelis, Miribel, France) was equipped with a flat membrane $(167 \mathrm{~mm} \times 75 \mathrm{~mm}$, effective surface area $0.0125 \mathrm{~m}^{2}$ ). The liquid channel was $0.5 \mathrm{~mm}$ thick with a free liquid vein (no spacer) for the run $1^{*}$ and $1.5 \mathrm{~mm}$ thick liquid vein with a polypropylene 46 mil feed spacer (1.168 mm thickness) placed inside as a turbulence promoter for all other test runs. 
The instantaneous permeation flow rate was computed from the permeate masses measured using a digital balance (RADWAG, WLC 6/A2) connected to a personal computer fitted with a data acquisition software. EPS solution was pumped into the membrane by a displacement pump (Quattroflow-1200 S, 4-piston diaphragm pump) and the feed solution pressure was adjusted with a throttling valve. Each experiment was carried out with a 2 L EPS solution volume which was continuously stirred and thermostatically controlled using a cryothermostat (Fisher Bioblock Scientific, Refrigerant / Heating Circulating Bath). Experiments were done at 20 or $40^{\circ} \mathrm{C}$. In the latter case, as well as during cleaning assays at $49{ }^{\circ} \mathrm{C}$ (see section 2.3.2 membrane cleaning), a hotplate stirrer (VELP Scientifica, AREX CerAlTop ${ }^{\mathrm{TM}}$ ) connected to a VTF digital thermoregulator (VELP Scientifica) was used. Temperatures of both permeate and retentate were monitored by thermocouples. Two pressure gauges were placed at the input and the exit of the membrane module in order to estimate the transmembrane pressure TMP as:

$$
T M P=\frac{P_{f}+P_{r}}{2}-P_{p}
$$

where $\mathrm{P}_{f}, \mathrm{P}_{\mathrm{r}}$ and $\mathrm{P}_{\mathrm{p}}$ (bar) are respectively the feed, retentate and permeate pressure, the latter being assumed constant.

\section{Insert Figure 1 here}

\subsubsection{Membrane conditioning and cleaning}

Membrane conditioning. Membrane samples were conditioned before the first use in order to remove preservatives (glycerine and sodium metabisulfite) and to obtain a reference water flux. The membrane was therefore soaked overnight (15 h) in deionized water, then water was filtered under 0.5 bar at a $1 \mathrm{~m} . \mathrm{s}^{-1}$ cross-flow velocity until $0.2 \mathrm{~L}$ of permeate was recovered. Thereafter, the membrane underwent an acid-alkaline cleaning (same procedure as in the membrane chemical cleaning). The membrane was then compacted at $20^{\circ} \mathrm{C}$ and a TMP of 2 bar until a stable water flux was reached. The water permeability of the pristine membrane was then measured. 
Membrane cleaning. After each experiment, the membrane was systematically rinsed with deionized water (6 L) at $27-29{ }^{\circ} \mathrm{C}$ in a non-recirculation mode (permeate and retentate flushed to drain) before chemical cleaning to remove any remaining build-up. The pure water flux was then measured in order to evaluate the irreversible part of the fouling.

Formulated Clean-In-Place (CIP) solutions were provided by Ecolab (France). The membrane was always cleaned with $2 \mathrm{~L}$ of the appropriate cleaning solution in a

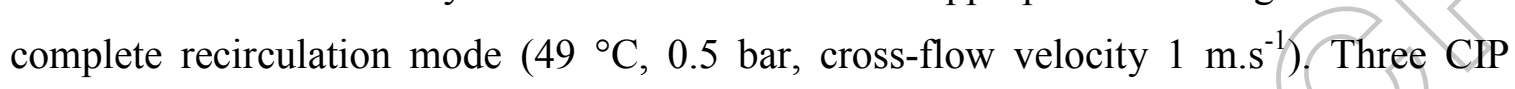
solutions were tested within different sequences (cf. part 3). Acidic and alkaline cleaning was performed for 20 mins respectively with P3-Ultrasil 75 solution $(0.104 \mathrm{v} / \mathrm{w} \%$ in water, $\left.49{ }^{\circ} \mathrm{C}, \mathrm{pH} 2\right)$ and P3-Ultrasil 110 solution $\left(0.223 \mathrm{v} / \mathrm{w} \%\right.$ in water, $\left.49^{\circ} \mathrm{C}, \mathrm{pH} 11\right)$. The enzymatic solution was a mixture of the buffer P3-Ultrasil $53(0.8 \mathrm{w} / \mathrm{v} \%$ in water, 49 ${ }^{\circ} \mathrm{C}, \mathrm{pH} \sim 9.15$ ) and the combination of enzymes and surfactants P3-Ultrasil 62A. P3Ultrasil 53 was first circulated for 10 mins before P3-Ultrasil $62 \mathrm{~A}$ was added at $0.5 \mathrm{w} / \mathrm{v} \%$ and the mixture circulated for 40 mins. Right after each CIP, the pilot plant was thoroughly rinsed with water and the cleaning efficiency was evaluated by comparing the water flux recovery to that of the pristine membrane. The hydraulic cleanliness was considered to be achieved when at least $90 \%$ of the initial water flux was recovered.

\subsubsection{Ultrafiltration of EPS solutions}

Table 3 summarizes the different experimental runs performed in this study and their operating conditions. All ultrafiltration (UF) runs were carried out in a batch mode with a complete recirculation of both retentate and permeate to the feed tank in order to maintain a constant concentration in EPS (VRR of 1). All runs were performed with a 1.5 $\mathrm{mm}$ thick fluid vein equipped with a spacer excepted run $1^{*}$ where a free, $0.5 \mathrm{~mm}$ thick fluid vein was used. For the fouling experiments, samples of permeate (taken directly from the outlet of the permeate pipe) and retentate (from the feed tank) were collected at each steady-state pressure step and stored at $-20{ }^{\circ} \mathrm{C}$ until further analyzes. Samples taken from the feed tank were also used to check if the EPS concentration remained constant during the complete recirculation mode. 
Filtration tests were carried out at $20^{\circ} \mathrm{C}$ for all runs except for run 13 performed at $40{ }^{\circ} \mathrm{C}$. Water fluxes were systematically normalized to 20 or $40{ }^{\circ} \mathrm{C}$ to smooth the impact of temperature and viscosity fluctuations on permeation fluxes.

\subsubsection{Determination of limiting and critical permeation fluxes}

Establishment of the J-TMP curves. The results of the filtration tests are presented by plotting the steady-state permeate flux $J$ against the transmembrane pressure TMP.Each point on the curves represents the steady state flux value at a given TMP (see Figures 2,5 and 7). The TMP was increased stepwise from the minimum adjustable pressure on the pilot (which increases with the tangential velocity) until the region where the flux independent of the TMP was reached. For each value of TMP, the evolution of the permeate flux $\mathrm{J}$ over time was followed and the value of the steady-state flux was measured after the flux had leveled off.

Limiting flux $J_{\text {lim }}$ for a given tangential velocity $V$. The limiting flux $\mathrm{J}_{\lim }$ is the maximum stationary permeation flux which can be reached when increasing TMP $[21,22]$. $\mathrm{J}_{\lim }$ correspond to a flux for which fouling saturates the filtration capacity of the membrane [22]. The TMP at which $\mathrm{J}_{\text {lim }}$ is obtained is called the limiting TMP and noted TMP $\mathrm{P}_{\text {lim }}$. Each filtration was stopped as soon as $\mathrm{J}_{\mathrm{lim}}$ was reached in order not to exceed the limiting pressure and not to let the membrane operate for a long time in strongly fouling conditions.

Nominal transmembrane pressure TMP ${ }_{n}$. TMP $\mathrm{n}_{\mathrm{n}}$ was defined as the TMP for which the permeation flux is equal to $75 \%$ of the limiting flux $\mathrm{J}_{\text {lim }}$, a value taken as an estimate of the critical flux $J_{\text {ci. }}$.

Determination of the critical flux for irreversibility $J_{c i}$. The critical flux for irreversibility was valued following the method of square wave of applied pressure proposed by [23, 24] for evaluating the reversibility of colloidal fouling layers. The method was used with the solution B $\left(0.16 \mathrm{~kg} \mathrm{GlcEq} \cdot \mathrm{m}^{-3}\right)$ in a complete recirculation mode $(\mathrm{VRR}=1)$ with a fixed cross-flow fluid velocity of $1.0 \mathrm{~m} \cdot \mathrm{s}^{-1}$ at $20{ }^{\circ} \mathrm{C}$. The principle of the method is explained in section 3.3.2. 


\subsubsection{Resistance in series analysis}

Deionized water was filtered at the beginning of each experiment to enable the clean membrane resistance $R m$ to be calculated according to Darcy's law:

$$
J_{W 0}=\frac{T M P}{\eta \times R_{m}}=L p_{w 0} \times T M P
$$

where $\mathrm{JW}_{0}$ is the permeate flux $\left(\mathrm{m} \cdot \mathrm{s}^{-1}\right.$ or $\left.\mathrm{L} \cdot \mathrm{h}^{-1} \cdot \mathrm{m}^{-2}\right), \eta$ the permeate (assimilated to pure water) dynamic viscosity (Pa.s), $R_{m}$ the membrane hydraulic resistance $\left(\mathrm{m}^{-1}\right)$, TMP the transmembrane pressure (bar) and $\mathrm{Lp}_{\mathrm{w} 0}$ the water permeability $\left(\mathrm{m} . \mathrm{s}^{-1}\right.$. bar ${ }^{-1}$ or $\mathrm{L} . \mathrm{h}^{-1} \cdot \mathrm{m}^{-}$ $\left.{ }^{2} \cdot \mathrm{bar}^{-1}\right)$.

The additional fouling resistances that appeared during the filtration of EPS were evaluated according to the "Resistance-in-Series" model according to the following equation:

$$
J=\frac{T M P}{\eta \times R_{t}}=\frac{T M P}{\eta \times\left(R_{m}+R_{f}\right)}=\frac{T M P}{\eta \times\left(R_{m}+R_{\text {rev }}+R_{\text {irrev }}\right)}
$$

where $R_{t}$ is the total hydraulic resistance, $R_{f}$ the overall fouling resistance, $R_{\text {rev }}$ the reversible part of $R_{f}$ that is eliminated by a simple water rinsing and $R_{\text {irrev }}$ the irreversible fouling requiring chemical cleaning to be removed. $\eta, R_{m}$ and TMP are the same as in equation (2). The values of the resistances were measured experimentally as followed: $R_{m}$ by a water flux measurement on a clean membrane prior to each run (eq. 2); $\mathrm{R}_{\mathrm{t}}=\left(\mathrm{R}_{\mathrm{m}}+\right.$ $\left.R_{f}\right)$ with the stabilized permeate flux measurements at the end of the run (eq. 3); $\left(R_{m}+\right.$ $R_{\text {irrev }}$ ) with water flux measurements after water rinsing (physical cleaning) and $R_{\text {rev }}$ as the difference between $R_{\mathrm{f}}$ and $\mathrm{R}_{\text {irrev }}$.

\section{Results and discussion}

The aim of this study is to evaluate the filterability of $P$. cruentum EPS solutions by modules equipped with organic, PES membranes. The cleaning protocol of the membrane is first assessed (3.1), then the influence of tangential velocity $\mathrm{V}$, transmembrane pressure TMP, EPS concentration $c_{0}$ and temperature $\mathrm{T}$ on permeate 
fluxes are evaluated in a complete recirculation mode (section 3.2). Finally, the sensitivity of membrane fouling to the operating parameters (section 3.3) and the feasibility of EPS isolation by organic, PES membrane at an industrial level (3.4) are discussed.

EPS from Porphyridium sp. having high molecular weight, a membrane with 50 $\mathrm{kDa}$ MWCO was selected to ensure a complete retention of EPS and avoid pore clogging. Although they are susceptible to consequential fouling by adsorption of proteins and other organic molecules due to their hydrophobic character, PES membranes have several advantages (low investment costs, good thermal stability and chemical resistance, see Cheryan [25]) that make them regularly used in the industry in spiral wound modules for the ultrafiltration of biological fluids such as skim milk and whey [26, 27].

\subsection{Cleaning protocol assessment}

As several flux-PTM curves had to be established up to the limiting flux with the same membrane, this one would work in highly fouling conditions. Therefore, an effective cleaning protocol had to be determined in order to clean correctly the membrane and recover its permeability over several cycles. Superficial velocity in spiral wound modules with feed spacer is in the range of $0.2-0.3 \mathrm{~m} . \mathrm{s}^{-1}[28,29]$. According to the recommendations of the flat module manufacturer, the first filtration run and the membrane cleaning tests were performed with a thin $0.5 \mathrm{~mm}$ thick fluid vein with no spacer, and at a fluid velocity of $1 \mathrm{~m} . \mathrm{s}^{-1}$ for which the flux-TMP relation was intended to be close the one with a $1.5 \mathrm{~mm}$ thick vein with a spacer. The efficiency of the cleaning protocol was assessed with the EPS solution $\mathrm{S}_{\mathrm{A}}$ at $20^{\circ} \mathrm{C}$ (run $1 *$ in Table 3 ).

The limiting flux $\left(21.6 \mathrm{~L} \cdot \mathrm{h}^{-1} \cdot \mathrm{m}^{-2}\right)$ was quickly reached even at $0.5 \mathrm{bar}$, the lowest TMP possible at a velocity of $1 \mathrm{~m} . \mathrm{s}^{-1}$ (the J-TMP curve was very similar to that for $\mathrm{V}=$ 0.3 m.s ${ }^{-1}$ in Figure 2). An alkaline-acid CIP was first tested allowing only $83 \%$ of the initial water flux to be recovered which was insufficient. Either the repetition of the alkaline cleaning step or the cleaning by sodium hypochlorite (150 ppm with P3- Ultrasil $110, \mathrm{pH}=11)$ failed to regenerate the membrane. Enzymatic cleaning was thus tested on a second membrane $\mathrm{MQ}_{2}$ after the filtration of the same EPS solution $\mathrm{S}_{\mathrm{A}}$, but in usual hydrodynamic conditions in industrial spiral wound module, i.e. with a cross-flow 
velocity of $0.3 \mathrm{~m} \cdot \mathrm{s}^{-1}$ and a fluid vein of $1.5 \mathrm{~mm}$ width containing a 46 mil spacer (run 1). The limiting flux $\mathrm{J}_{\mathrm{lim}}, 20.7 \mathrm{~L} \cdot \mathrm{h}^{-1} \cdot \mathrm{m}^{-2}$, was reached rapidly for a low TMP of 0.5 bar (see the flux-TMP curve for $\mathrm{V}=0.3 \mathrm{~m} \cdot \mathrm{s}^{-1}$ in Figure 2), and was very close to that of run 1*, meaning that filtration performances are indeed very close in the operating conditions "V $=1 \mathrm{~m} \cdot \mathrm{s}^{-1}$ in a thin, free fluid vein" and "V $=0.3 \mathrm{~m} \cdot \mathrm{s}^{-1}$ with a spacer in the fluid vein".

Otherwise, the membrane was correctly regenerated by a three-steps cleaning procedure using successively the enzymatic mixture, acid cleaning and alkaline cleaning. The cleaning efficiency was ensured almost entirely by the first step of enzymatic cleaning meaning that EPS are involved in the irreversible fouling of the membrane. The acid and alkaline cleaning steps were nevertheless kept to inactivate the enzyme and to recondition the membrane, respectively. After this CIP procedure, the membrane was regenerated and the water permeability of the membrane was increased by about $25 \%$ in comparison with that measured on the brand new membrane without any filtration. Li et $a l$. [14] reported that an alkaline cleaning can properly regenerate a PES membrane when microalgal EPS from different strains were filtered below the critical flux. The enzyme cleaning step is necessary in the present work most probably because the membrane works under strongly fouling conditions. However, it cannot be excluded that the EPS solutions filtered here have a higher fouling capacity that those in the work of $\mathrm{Li}$ et al., possible causes being higher molecular weights of EPS and/or the presence of small organic compounds that could block the pores. In particular, the EPS solutions in the work of Li et al. [14] were filtered at $0.3 \mu \mathrm{m}$ and ours, no, so that they contain probably less colloidal matters.

\section{Insert Table 3 here}

\section{Insert Figure 2 here}

\subsection{Influence of operating parameters on EPS filtration}

3.2.1. Cross-flow velocities $V$ and transmembrane pressure TMP

Transmembrane pressure TMP and tangential velocity $\mathrm{V}$ that condition flow pattern (Reynolds number) and wall shear stress are usually the main parameters 
governing both the energetic requirements of a membrane filtration and the fouling of the membrane. Therefore, flux-TMP curves at different velocities $\mathrm{V}$ are a useful tool to evaluate the feasibility of a membrane operation. The curves were established for the filtration of the EPS solution $\mathrm{S}_{\mathrm{A}}\left(0.10 \mathrm{~kg} \mathrm{GlcEq} \cdot \mathrm{m}^{-3}\right)$ in the following conditions: a fluid vein $1.5 \mathrm{~mm}$ width with a feed spacer, temperature $20^{\circ} \mathrm{C}$, tangential velocity $\mathrm{V}: 0.3,0.6$, 1.0 and $1.2 \mathrm{~m} . \mathrm{s}^{-1}$ (runs $1-4$ ).

For a given TMP, an increase in the fluid velocity $\mathrm{V}$ always led to an increase of the steady state permeation flux whatever the pressure. For a given velocity, an increase in the TMP firstly led to an increase in flux, but then a plateau was reached at the socalled limiting flux, $\mathrm{J}_{\lim }$. An excessive increase in TMP seems to induce a decrease in permeation flux. As explained by Jönsson and Trägårdh [30], the decrease in permeation flux at high pressures can be due either to the compression of the formed cake or gel layer or to the compaction of the polymeric membrane itself. Such a phenomenon has already been observed with biological suspensions containing polysaccharides such as suspensions of microalgae or bacteria (see for instance Rossi et al. [16]). As mentioned earlier, for $\mathrm{V}=0.3 \mathrm{~m} \cdot \mathrm{s}^{-1}$, a typical value in spiral wound modules, the limiting flux $\mathrm{J}_{\lim }=$ 20.7 L.h $\mathrm{h}^{-1} \cdot \mathrm{m}^{-2}$ was reached rapidly at a low TMP of 0.5 bar (see Figure 2). Thereby, it seems that the EPS solution fouled strongly the membrane under these conditions.

As the nominal TMP was already low $(\sim 0.25$ bar $)$ for this experiment at $0.10 \mathrm{~kg}$ GlcEq. $\mathrm{m}^{-3}$, it turned out that it would be even lower for more concentrated EPS solutions, so that the reachable concentration factor in a UF operated in a concentration mode would probably be very limited.. Thus, to isolate and concentrate the P. cruentum EPS with an organic PES membrane, it will be probably necessary to work in more favorable hydrodynamic conditions by increasing the tangential velocity V. Therefore, the TMPflux curves have been established for 3 other values of $\mathrm{V}$ ranging from 0.6 to $1.2 \mathrm{~m} . \mathrm{s}^{-1}$ (Figure 2). As expected, the limiting permeate flux $\mathrm{J}_{\mathrm{lim}}$ increased with the fluid velocity from $20.7 \mathrm{~L} \cdot \mathrm{h}^{-1} \cdot \mathrm{m}^{-2}$ at $0.3 \mathrm{~m} \cdot \mathrm{s}^{-1}$ to $60 \mathrm{~L} \cdot \mathrm{h}^{-1} \cdot \mathrm{m}^{-2}$ at $1.2 \mathrm{~m} \cdot \mathrm{s}^{-1}$ ). So, higher shear stresses resulting from higher velocities reduce the thickness of the concentration boundary layer and prevent the accumulation of materials on the membrane surface [27]. Otherwise, the evolution of $\mathrm{J}_{\text {lim }}$ against $\mathrm{V}$ fit very well the "gel layer model" proposed by Blatt et al. [31] to explain the TMP independence of flux in ultrafiltration when the TMP is increased 
beyond a certain value. The gel layer model results from the film theory in the case where (i) the solute concentration on the membrane surface is high enough that it undergoes a gelation (or more generally, any liquid-solid phase transition) and (ii) the solute concentration in the permeate is negligible. The limiting flux is then linked to the solute concentration in the bulk by the relation:

$$
J_{\text {lim }}=k \ln \frac{C_{g}}{C_{0}}
$$

where $\mathrm{C}_{\mathrm{g}}$ is the gel concentration of the solute and $\mathrm{k}$ the mass transfer coefficient in the polarization layer which can be expressed with respect to the tangential yelocity $V$ using a mass transfer correlation. When a set of experiments are carried out with the same solution and at the same temperature, the gel model leads to the following relation between the limiting flux $J_{\lim }$ and the tangential velocity $V$ (see Appendix 1):

$$
\mathrm{J}_{\lim }=\mathrm{C} \times \mathrm{V}^{a}
$$

where $\mathrm{C}$ and $a$ are 2 constants. Figure 3 shows that experimental results fit quite well the proportional relationship between $\mathrm{J}_{\lim }$ and $\mathrm{V}$ (coefficient of determination $\mathrm{R}^{2}=0.99$ ) with $a=0.77$. This value is fully coherent with those for turbulent flow, which are known to be comprised between 0.75 and 0.90 [32], meaning that nothing obviously limits the positive influence of the fluid velocity on the limiting flux.

\section{Insert Figure 3 here}

Flux-TMP curves were also used to choose the tangential velocity $\mathrm{V}$ for the runs concerning the influence of the concentration in EPS $c_{0}$ on permeation flux and membrane fouling by taking into account the extend of the pressure drop across the module. As $\mathrm{V}$ increased, fouling decreased and permeate fluxes increased, but so did the pressure drop across the module. Such high value increases the minimum TMP usable and makes the membrane work more heterogeneously. It would also increase pumping costs on an industrial plant. Thus, pressure drop was $0.04,0.14,0.27$ and 0.36 bar for 0.3 , $0.6,1.0$ and $1.2 \mathrm{~m} . \mathrm{s}^{-1}$ respectively at the minimal accessible TMP and for a membrane length of $16.8 \mathrm{~cm}$. For this reason, a cross-flow velocity of $1.0 \mathrm{~m} . \mathrm{s}^{-1}$ seemed to be a reasonable compromise and was thus selected for all subsequent experiments. 
By the end, a filtration was carried out in the same conditions of those of run 3 but at a single TMPn of 0.57 bar representing the nominal TMP for a $1.0 \mathrm{~m} \cdot \mathrm{s}^{-1}$ tangential velocity (run No. 5). The stabilized permeation flux obtained under this TMP $_{n}$ was 41.2 L. ${ }^{-1} \cdot \mathrm{m}^{-2}$, which is very close to that in run $3\left(40.8 \mathrm{~L} \cdot \mathrm{h}^{-1} \cdot \mathrm{m}^{-2}\right)$ for the same TMP. The agreement between the two values can be interpreted as a clue of the stability of the membrane hydraulic resistance $\mathrm{R}_{\mathrm{m}}$ after regeneration (this will be confirmed by the examination of the fouling resistances in section 3.3.1). Figure 4 shows the evolution of the permeate flux $\mathrm{J}$ over time for the filtration. During the filtration at the nominal TMP (Figure $4 \mathrm{~b}$ ), the flux exhibits a sharp drop during the first 20 min flux followed by a slower decline before reaching a steady-state value after about 170 minutes. Flux stabilization was much faster for experiments where TMP was increased step by step until the limiting flux is obtained, with a typical duration of about 25 to $50 \mathrm{~min}$ (see Figure 4a).

\section{Insert Figure 4 here}

\subsubsection{EPS concentration $c_{0}$}

The effect of EPS concentration on fouling experiments was studied through the filtration of the 4 solutions $\mathrm{S}_{\mathrm{A}}, \mathrm{S}_{\mathrm{B}}, \mathrm{S}_{\mathrm{Bcd} 1}$ and $\mathrm{S}_{\mathrm{Bcd} 2}$ with respective EPS concentrations of $0.10,0.16,0.64$ and $1.06 \mathrm{~kg} \mathrm{GlcEq. \textrm {m } ^ { - 3 }}$ (runs 1, 6, 7 and 8, 9; membrane sample $\mathrm{MQ}_{2}$, cross-flow velocity $1.0 \mathrm{~m} \cdot \mathrm{s}^{-1}$, temperature $20^{\circ} \mathrm{C}$, VRR 1 ). The filtration of solution $\mathrm{S}_{\mathrm{Bcd} 1}$ was performed twice, without and with a clarification by centrifugation (runs 7 and 8 , respectively) to remoye precipitates that appeared in small quantities after thawing. As no difference was observed between the two flux-TMP curves, it was considered thereafter that the presence of these precipitate did not lead to any bias.

EPS retention. The EPS concentration in permeates was very low and below the threshold of quantification for all solution concentrations $c_{0}$, and even undetectable. Hence, the retention in EPS by the membrane can be considered total as expected because of the MWCO of the membrane.

Permeation fluxes. The filtration curves for the various concentrations are shown on Figure 5. As the EPS concentration $c_{0}$ in the feed solution increased, the limiting 
permeate flux $\mathrm{J}_{\text {lim }}$ decreased and was reached for a lower TMP value (see Figure 5 and Table 4), because of the amount of additional material likely to foul the membrane. However, the decay in $\mathrm{J}_{\lim }$ when $\mathrm{c}_{0}$ increased did not seem to agree with the gel layer model. According to equation (4), the $\mathrm{J}_{\lim }$ plot against $\mathrm{c}_{0}$ should intersect the abscissa axis at $\log \left(c_{g}\right)$. Now the plot here gives a $c_{g}$ value of $47 \mathrm{~kg}$ GlcEq. $\mathrm{m}^{-3}$ which seems to be high (see Figure 6). Indeed, Balti et al. [33] estimated a $\mathrm{c}_{\mathrm{g}}$ value of around $5.5 \mathrm{~kg}$ GlcEq. $\mathrm{m}^{-3}$ when they concentrated a supernatant of P. cruentum with an inorganic microfiltration membrane (mean pore diameter $0.14 \mu \mathrm{m}$ ) up to a VRR of 10. Cheryan [25] (section $4 \mathrm{G}$ ) indicates that the experimental values of $c_{\mathrm{g}}$ can be much lower than those predicted by the gel layer model if the solution viscosity and solutes diffusion coefficient vary greatly with the increase in $\mathrm{c}_{0}$, which is the case for highly viscous polysaccharide solutions [12, $13]$.

Therefore, the permeation fluxes that would be obtained during a concentration mode filtration up to a VRR of about 10 could be lower than those obtained here. Nevertheless, they indicate that $P$. cruentum EPS can probably be isolated from supernatants obtained outdoors in open circulating pond with quite high concentration factors. As already mentioned, Li et al. [14] reported that EPS of various photosynthetic microorganisms could be isolated from culture supernatants using a plate UF unit up to high volume reduction factors (20 and sometimes 40) with a mean permeate flux around $35 \mathrm{~L} \cdot \mathrm{h}^{-1} \cdot \mathrm{m}^{-2}$. Although they didn't specify the EPS concentration $\left(\mathrm{c}_{0}\right)$ in the surpernatants processed, the EPS yields they mentioned allow to estimate that it ranged between 0,02 and $0,2 \mathrm{~kg} \cdot \mathrm{m}^{-3}$

\section{Insert Figure 5 here}

Insert Table 4 here

Insert Figure 6 here 


\subsubsection{Temperature $T$}

Temperature has a direct effect on permeate flux through the solvent viscosity but it can also affect fouling depending on feed solution and solute characteristics. So, the same EPS solution $\left(\mathrm{S}_{\mathrm{Bcdl}}, 0.64 \mathrm{~kg}\right.$ GlcEq. $\left.\mathrm{m}^{-3}\right)$ was filtered in the same conditions but at two different temperatures in order to evaluate the temperature effect on the filterability. The limiting permeate flux $J_{\text {lim }}$ was found to increase by $52 \%$ from 33.9 to $51.5 \mathrm{~L} . \mathrm{h}^{-1} \cdot \mathrm{m}^{-2}$ when the temperature rose from 20 to $40{ }^{\circ} \mathrm{C}$ (runs $12 \& 13$ ).

The literature reports four different potential influences of temperature on permeate flux. According to Jönsson and Trägårdh [30] and Cheryan [25] (section 6.D.3), (i) an increase in temperature reduces the viscosity of the solution and thus increases the permeate flux according to Darcy's law. On the other hand, (ii) the rise in permeate flux can increase the accumulation of solutes at the membrane wall and thus the solution osmotic pressure, partly limiting the flow rise. Additionally, temperature variations can also (iii) decrease the solubility of some inorganic compounds such as calcium phosphate (calcium and phosphate ions being present in the P. cruentum culture medium). Furthermore, (iv) it may affect the physicochemical characteristics of macromolecules and their adsorption to the membrane surface and can therefore have an effect on the solute retention efficiency [34]. In our case, cause (ii) can be ruled out as the major solutes are high molecular weight EPS, as well as cause (iv) because of the total retention of EPS by the $50 \mathrm{kDa}$ MWCO membrane. In order to evaluate the direct influence of T on flux through the decrease in fluid viscosity (cause i), the permeate fluxes measured at $40{ }^{\circ} \mathrm{C}$ were corrected at $20{ }^{\circ} \mathrm{C}$ assuming the permeate had the same viscosity as pure water. The corrected flux-TMP curve was then compared to that measured directly at 20 ${ }^{\circ} \mathrm{C}$. It can be seen in Figure 7 that the flux-PTM measured à $20{ }^{\circ} \mathrm{C}$ and the one established at $40{ }^{\circ} \mathrm{C}$ but corrected to $20^{\circ} \mathrm{C}$ are virtually superimposable, meaning that the influence of $\mathrm{T}$ on permeation fluxes can be totally explained by the viscosity decrease.

To conclude, the results indicate that a filtration at $40{ }^{\circ} \mathrm{C}$ would be appropriate when using a MWCO low enough for the membrane retaining totally the EPS. A similar conclusion was reported by Li et al. [14] who isolated EPS from the cell-free medium of H. pluvialis clarified by microfiltration using an ultrafiltration PES membrane with a 5 
kDa MWCO. The authors found that the permeate flux increased from 33.9 to 57.7 L.h ${ }^{-}$ ${ }^{1} . \mathrm{m}^{-2}$ (70\% increase) when the temperature rose from $20^{\circ} \mathrm{C}$ to $50^{\circ} \mathrm{C}$, and they concluded that a temperature between 30 and $40{ }^{\circ} \mathrm{C}$ was appropriate to isolate EPS by ultrafiltration.

\section{Insert Figure 7 here}

\subsection{Fouling characterization}

The nature and extent of fouling during the filtration of EPS solutions were characterized firstly on the basis of the hydraulic resistances of reversible and irreversible fouling, then using the square wave procedure for determining the eritical flux for irreversibility.

\subsubsection{Hydraulic resistances of membrane and fouling layer}

Figure 8 displays the resistances values of the membrane $\left(\mathrm{R}_{\mathrm{m}}\right)$ and the reversible $\left(\mathrm{R}_{\text {rev }}\right)$ and irreversible $\left(\mathrm{R}_{\text {irrev }}\right)$ parts of fouling for all the test runs carried out on the membrane samples $\mathrm{MQ}_{2}$ and $\mathrm{MQ}_{3}$. Runs are numbered chronologically in the order they were performed. $R_{m}$ was measured prior to each experiment and $R_{\text {rev }}$ and $R_{\text {irrev }}$ at the end of the procedure. Note that all the runs excepted No. 5 consisted in establishing the fluxPTM curve by a step-by-step increase in TMP. Consequently, the fouling resistances $\mathrm{R}_{\text {rev }}$ and $\mathrm{R}_{\text {irrev }}$ are not associated with specific hydrodynamics conditions but are rather the cumulative result of successive filtrations carried out at several increasing pressures until the limiting flux was reached, meaning that the membrane worked in severe fouling conditions. Therefore, fouling resistances and $\mathrm{R}_{\mathrm{rev}}$ in particular should be analyzed and compared with caution.

\section{Insert Figure 8 here}

The membrane resistance. $\mathrm{R}_{\mathrm{m}}$ decreased by $27 \%$ from $4.8 \times 10^{12}$ to $3.5 \times 10^{12} \mathrm{~m}^{-1}$ on the first 3 experiments (runs 1-3), after which $\mathrm{R}_{\mathrm{m}}$ reaminded stable at $\pm 5 \%$ for subsequent tests. This behavior was also observed with the membrane sample $\mathrm{MQ}_{3}$ (data not shown). As $R_{m}$ is independent of fouling and fluid flow conditions [27], the decrease in $R_{m}$ values after the chemical cleaning steps of the first runs can be attributed to a modification of 
membrane morphology or surface quality such aspores size, hydrophobicity or surface charge or the progressive formation of a residual fouling layer up to a stable state.

The influence of the cross-flow velocity on fouling was carried out on solution $\mathrm{S}_{\mathrm{A}}$ at a concentration in EPS of $0.10 \mathrm{~kg} \mathrm{GlcEq} \cdot \mathrm{m}^{-3}$ (runs 1-4). It can be seen in Figure 8 that the value of $R_{\text {irrev }}$ is roughly the same (about $2.2 \times 10^{12} \mathrm{~m}^{-1}$ ) regardless of the cross-flow velocity $\mathrm{V}$, the cleaned membrane resistance and the last TMP used ( 1 bar for $0.3 \mathrm{~m} . \mathrm{s}^{-1}$ but 1.5 bar for $0.6,1.0$ and $1.2 \mathrm{~m} . \mathrm{s}^{-1}$ ). Irreversible fouling is due to the deposition of solutes onto the external surface and/or within the pores of the membrane (adsorption, pore blockage, irreversible deposit or gel) which can be removed only by chemical cleaning. Under the operating conditions investigated in runs 1-4, it seems that the irreversible fouling was quite independent of the cross-flow velocity contrary to the reversible fouling. Indeed, $\mathrm{R}_{\text {rev }}$ decreased when the cross-flow velocity increased, expressing the positive influence of this parameter on reversible fouling due to the increase in the lift force by greater wall shear stress that limited the solutes deposition on the membrane.

In any case, the resistance of the reversible fouling $\left(\mathrm{R}_{\text {rev }}\right)$ in runs 1-4 was greater than that of the irreversible fouling ( $\left.\mathrm{R}_{\mathrm{irrev}}\right)$, but as explained in the first paragraph of this part, the fact should be interpreted with caution. In that respect, comparing runs 3 and 5 indicates that the irreversible resistance was higher than the reversible one in run 5 (moderate fouling conditions) contrary to what was observed for the run 3 (same tangential velocities but more severe fouling conditions). For the filtration under nominal TMP at $1.0 \mathrm{~m} . \mathrm{s}^{-1}$ (run 5), $\mathrm{R}_{\text {irrev }}$ is significantly lowered but amounts nevertheless to $88 \%$ of the overall fouling resistance. So, in moderate fouling conditions and with low EPS concentrations, the irreversible fouling is the main cause of flux decrease whereas the part of reversible fouling becomes predominant when approaching the limiting flux.

Regarding experiments on the influence of EPS concentration, reversible and irreversible fouling resistances were found to keep almost the same value for all the filtrations of solutions prepared from the supernatant $\mathrm{B}$ (runs 6-9, $\mathrm{R}_{\text {rev }}$ and $\mathrm{R}_{\text {irrev }} \approx 2.8$ and $0.9 \times 10^{12} \mathrm{~m}^{-1}$ respectively) while EPS concentrations $\mathrm{c}_{0}$ increased by a factor of 6.6 $\left(0.16\right.$ to $\left.1.06 \mathrm{~kg} \mathrm{GlcEq} \cdot \mathrm{m}^{-3}\right)$. One possible reason is that the maximum TMP imposed on 
the plateau decreased when the concentration of EPS was increased (see Figure 5) so that the opposite influences of the two parameters on the fouling compensate each other. On the other hand, the irreversible fouling resistances for the filtrations of solutions $\mathrm{B}, \mathrm{B}_{\mathrm{cd} 1}$ and $\mathrm{B}_{\mathrm{cd} 2}\left(\mathrm{R}_{\text {irrev }} \sim 0.9 \times 10^{12} \mathrm{~m}^{-1}\right)$ were lower than those for the filtration of solution $\mathrm{S}_{\mathrm{A}}$. This hold even for the run $5\left(\mathrm{R}_{\text {irrev }}=1.5 \times 10^{12} \mathrm{~m}^{-1}\right.$, run 5$)$ carried out a priori in less fouling conditions (same velocity but nominal TMP and lower EPS concentration). One possible explanation is that the organic matter of solution A has a fouling capacity greater than that of solution $\mathrm{B}$. Indeed, the $\mathrm{OM}$ excreted by microalgae depends on the cultivation conditions (solar flux and temperature in particular) and the culture age [5]. The fact that culture A was older than culture B (resp. 22 and 18 days) is compatible with the hypothesis.

Finally, both the irreversible and reversible resistances at the plateau are almost identical at 20 and $40{ }^{\circ} \mathrm{C}$, confirming that $\mathrm{T}$ has little or no impact on fouling establishment as hypothesized in section 3.2.3.

\subsubsection{Estimation of the critical flux for irreversibility $J_{c i}$}

Before estimating the critical flux for irreversibility, we sought whether the critical flux was of weak or strong form, i.e. if there is a rapid adsorption of material at the beginning of the filtration or not. The decrease in permeability (J/TMP) between the clean water flux measurement just before the filtration and the filtration of the EPS solution at low TMP was determined for the 4 tests carried out with the solution $\mathrm{S}_{\mathrm{A}}$. The decrease was clear but moderate with an average value of $20.5 \pm 4.0 \%$ which indicates the existence of an additional flow resistance during the filtration of EPS solutions at low TMP. The cause may a priori be either a moderate adsorption or the formation of a polarization layer. Indeed, the increase in EPS solutions viscosity with the EPS content even at low concentration could reduce the back transfer of EPS accumulated near the membrane surface. Therefore, the issue of the existence of a moderate adsorption cannot be solved without a rheological study of filtered EPS solutions and a study of the flow of these solutions near the membrane surface $[12,13]$.

The critical flux for irreversibility $\mathrm{J}_{\mathrm{ci}}$ was defined by Bacchin et al. [22] as the flux above where an irreversible fouling of the membrane appears. Note that the concept 
of reversibility considered by these authors is not the one used here. In our paper, the reversible resistance is that of the part of the solid deposit on the membrane which will be eliminated by sweeping it with water after stopping the filtration of the solution, the irreversible resistance being that of the residual part whose elimination requires a chemical cleaning of the membrane. For Bacchin et al. [22], the reversible resistance to permeation is the one which will gradually disappear when the pressure is decreased during the filtration of the solution. The reversible resistance is offered by molecules or particles accumulated more or less compactly in the vicinity of the fouled membrane but without significant interaction with the deposit already formed on the membrane surface, whereas the irreversible resistance is that of the deposit already formed. To mark the difference between the two notions of reversibility, an asterisk will be attached to that of Bacchin et al. [22].

$\mathrm{J}_{\mathrm{ci}}$ was determined by filtering the solution $\mathrm{S}_{\mathrm{B}}\left(0.16 \mathrm{~kg}\right.$ GlcEq.m $\left.\mathrm{m}^{-3}\right)$ according to the procedure proposed by Espinasse et al. [24]. The method consists in imposing successive square waves of pressure composed each one of an upper (U) and a lower (L) pressure step while continually monitoring the permeate flux J. For example, the first 2 square waves in Figure $9 \mathrm{a}$ are $\left(\mathrm{U}_{1}, \mathrm{~L}_{1}\right)=(\mathrm{b}, \mathrm{c})$ and $\left(\mathrm{U}_{2}, \mathrm{~L}_{2}\right)=(\mathrm{d}, \mathrm{e})$. Two successive square waves $\left(\mathrm{U}_{\mathrm{n}-1}, \mathrm{~L}_{\mathrm{n}-1}\right)$ and $\left(\mathrm{U}_{\mathrm{n}}, \mathrm{L}_{\mathrm{n}}\right)$ are such that $\mathrm{TMP}_{\mathrm{Un}}>\mathrm{TMP}_{\mathrm{Un}-1}$ and $\mathrm{TMP}_{\mathrm{Ln}}=$ $\mathrm{TMP}_{\mathrm{Un}-1}$, i.e. the pressure after the lower step $\mathrm{L}_{\mathrm{n}}$ is brought back to the upper pressure imposed at the previous wave (with an exception for the first square wave). The occurrence of a critical flux is then determined as follows. Firstly, the presence of a flux decline at an upper step $U_{n}$ indicates that the increase in TMP has increased the membrane fouling and that a kind of critical flux has been reached (for instance $\mathrm{J}_{\mathrm{d}}$ on Figure 9a). Then, the type of additional fouling appeared after step $U_{n}$ is diagnosed by comparing the stabilized permeate fluxes at the same pressure before and after the square wave (i.e., $\mathrm{J}_{\mathrm{Ln}}$ and $\mathrm{J}_{\mathrm{Un}-1}$ for $\mathrm{n}>1$ and $\mathrm{J}_{\mathrm{a}}$ and $\mathrm{J}_{\mathrm{c}}$ for the $1^{\text {st }}$ wave). The additional fouling appeared at step $U_{n}$ is considered as totally reversible if fluxes $J_{\mathrm{Ln}}$ and $\mathrm{J}_{\mathrm{Un}-1}$ are not significantly different, and partly irreversible otherwise.

\section{Insert Figure 9 here}


Here, TMP was firstly increased from 0.24 to 0.85 bar by 0.20 bar increments (Figure 9a). It can be seen that a critical flux was reached from the first upper step since a flux decline with time was observed at this pressure (step b, 0.45 bar). In addition, the stabilized flux after the first lower step c $(0.24$ bar $)$ was not totally recovered but decreased by $9.6 \%$ between steps a $\left(26.9 \mathrm{~L} \cdot \mathrm{h}^{-1} \cdot \mathrm{m}^{-2}\right)$ and c $\left(24.3 \mathrm{~L} \cdot \mathrm{h}^{-1} \cdot \mathrm{m}^{-2}\right)$. This means that the fouling was already (in part) irreversible and that the critical flux for irreversibility $\mathrm{J}_{\mathrm{ci}}$ was already exceeded at 0.45 bar. A second test run was thus carried out under the same conditions but with a smaller TMP increment of 0.10 bar (Figure 9b). Here again, after the first wave a'-b'-c', a small decrease between the stabilized fluxes $J_{\mathcal{c}^{\prime}}$, and $\mathrm{J}_{\mathrm{a}}$, was observed, meaning that an irreversible fouling existed already at 0.35 bar, e.g. that the critical flux is below $36 \mathrm{~L} \cdot \mathrm{h}^{-1} \cdot \mathrm{m}^{-2}$. The value of $\mathrm{J}_{\mathrm{ci}}$ was thus estimated by Espinasse et al. [24] by computing the total irreversible resistance at the upper steps as the sum of the additional resistances that appeared gradually at the $n$ first upper steps:

$$
\begin{gathered}
R_{\text {irrev }}^{*}\left(U_{n}\right)=\sum_{i \leq n} r_{\text {irrev }}^{*}\left(U_{i}\right) \\
r_{\text {irrev }}^{*}\left(U_{i}\right)=R_{f}\left(L_{i}\right)-R_{f}\left(U_{i-1}\right)
\end{gathered}
$$

where $r_{\text {irrev }}^{*}\left(U_{n}\right)$ is the additional irreversible resistance appearing at level $U_{n}$ and $R_{f}(S)$ the overall fouling resistance at step $S$. Plotting $R^{*}{ }_{\text {irrev }}$ against TMP allows then the estimation of the $\mathrm{TMP}_{\mathrm{ci}}$ pressure at which an irreversible fouling begins to manifest, i.e. at nil $\mathrm{R}_{\text {irrev. }}^{*}$ Here, a linear regression gives an estimation of $\mathrm{TMP}_{\mathrm{ci}}$ around $0.3 \mathrm{bar}$ (see Figure 10) which would correspond to a critical flux $\mathrm{J}_{\mathrm{ci}}=32 \mathrm{~L} \cdot \mathrm{m}^{-2} \cdot \mathrm{h}^{-1}$ and a ratio $\mathrm{J}_{\mathrm{ci}} / \mathrm{J}_{\mathrm{lim}}$ close to 0.6. However, the value of $\mathrm{J}_{\mathrm{ci}}$ is overestimated because the flux at the first upper step $U_{1}$ is not sub-critical therefore a part of the irreversible resistance was not taken into account in eq 6. Indeed, the high tangential velocity required for the EPS solution filtration results in a high pressure drop through the module, so that the minimal mean TMP is above the one corresponding to the critical flux. Similar values of $J_{c}$ have been already reported for the filtration on a PES $50 \mathrm{kDa}$ membrane of cells and 
macromolecules, i.e. $23 \mathrm{~L} \cdot \mathrm{m}^{-2} \cdot \mathrm{h}^{-1}$ for yeast and $32-55 \mathrm{~L} \cdot \mathrm{m}^{-2} \cdot \mathrm{h}^{-1}$ for BSA depending on Reynolds number (Wu et al. [35] cited by Bacchin et al. [22]).

\section{Insert Figure 10 here}

Finally, we would emphasize that the measurement of the critical flux could be hampered by a noticeable heterogeneity in the membrane permeability over its surface. RabillerBaudry [26] report three possible causes of such heterogeneity, namely (i) variations in permeability of the primary membrane, (ii) the axial gradient of TMP along the membrane, and (iii) the fluid distribution at the membrane inlet and the resulting local variations in fluid velocity and turbulence. Cause (i) is not considered here because it is not related to the hydrodynamics of the flow in the module, and cause (iii) not either as we have no insight on the fluid distribution at the module entry. Regarding cause (ii), the minimal TMP in this work was high as already mentioned because of the importance of the pressure drop in the module. For instance, in the condition of exp. 10 and 11 (see legend of Figure 9), the minimal TMP was 0.24 bar and thepressure drop $\Delta \mathrm{P}$ between the inlet and the outlet of the module 0.29 bar. The actual pressure drop along the membrane is lower because of the point losses at the module entrance and exit, but considering that the order of magnitude of the mean membrane permeability J/PTM at low TMP is around $100 \mathrm{~L} \cdot \mathrm{h}^{-1} \cdot \mathrm{m}^{-2} \cdot \mathrm{bar}^{-1}$, even a third of $\Delta \mathrm{P}$ would lead to a significant decrease in the permeate flux between the entrance and the exit of the module $\left(\sim 10 \mathrm{~L} \cdot \mathrm{h}^{-1} \cdot \mathrm{m}^{-2}\right)$ if the effect of the TMP gradient on flux was dominant. Furthermore, Cho and Fane [36] established from SEM examination that the fouling by EPS in membrane bioreactors (flat sheet MF membrane $0.22 \mu \mathrm{m}$ in hydrophilised PVDF) was progressively increasing from entry to the output of the module, which tends to further increase the ratio of the local flux of permeate between the inlet and the outlet due to the pressure drop. The authors indicated that 'the reason for this significant axial distribution of foulant is not clear but may be due to stronger eddies close to the inlet or the surface flow offoulant downstream due to shear'. 


\subsection{Feasibility of the extraction of EPS from P. cruentum supernatants by organic UF at an industrial scale}

Here the choice of filtration modules that could be used on an industrial scale to concentrate EPS solutions on organic membranes will be discussed.

P. cruentum culture media containing EPS are strongly fouling solutions. Table 5 compares the performance of the filtration of EPS solutions and of skim milk [26] carried out in similar conditions (same plate module, same operating mode VRR $=1$, similar UF membrane and close temperatures), the main difference being a much higher velocity in our work. It can be seen that limiting and critical fluxes $\mathrm{J}_{\lim }$ and $\mathrm{J}_{\mathrm{c}}$, respectively, were almost identical in both cases, this means that the fouling capacities of solutions are close to each other although the concentration in organic matter was much higher for skim milk filtration (ratio of about 1000) and the duration longer. As explained in part 3.3.2, the high fouling power of EPS solutions can be attributed to the high molar mass of EPS and their ability to form highly viscous gels and compact deposits on the membrane surface. The fact that $J_{\lim }$ and $J_{c}$ were obtained for higher pressures for skim milk should be related to the nature of critical flux. As indicated by the high drop between the permeabilities for pure water and the filtered solution at low pressures (resp. $\mathrm{L}_{\mathrm{pw} 0}$ and $\mathrm{L}_{\mathrm{p} 0}$ ), the membrane underwent a strong adsorption of organic matter during the filtration of skim milk. This corresponds clearly to the weak form of critical flux $\left(\mathrm{J}_{\mathrm{cw}}\right)$ according to Bacchin et al. [22], whereas the adsorption was clearly less pronounced during the filtration of EPS solutions.

Spiral wound (SW) modules were considered first because of their high compactness, low energy consumption and low system capital costs [25]. However, the results obtained in parts 3.2.1 and 3.3.2 show that when filtering the native EPS solution $\mathrm{S}_{\mathrm{A}}\left(\mathrm{c}_{0}=0.10 \mathrm{~kg} \mathrm{GlcEq} \cdot \mathrm{m}^{-3}\right)$ in a fluid vein with a feed spacer at a tangential velocity of $0.3 \mathrm{~m} . \mathrm{s}^{-1}$ typically encountered in spiral wound modules, the limiting flux was quickly reached even for a low TMP. Therefore, it can be expected that a high volume reduction ratio VRR could not be obtained in a SW module. Additional work is nevertheless needed to evaluate the possibility to pre-concentrate EPS solutions at a low VRR using a SW 
module before increasing the VRR on another module. Reaching high VRR in sustainable conditions would probably require working in more favorable hydrodynamic conditions by increasing the tangential velocity $\mathrm{V}$. This can be obtained either within a plate and frame (PF) module as has been previously done by Li et al. [14] or with a tubular module. An estimation of the wall shear stresses $\tau_{\mathrm{w}}$ in typical hydrodynamic conditions shows that they are significantly higher in both modules than in the spiral-wound one (resp. approximately $86 \mathrm{~Pa}$ and $58 \mathrm{~Pa}$ for a tubular module and a plate and frame module against $8 \mathrm{~Pa}$ in a spiral wound module, see Appendix 2).

\section{Insert Table 5 here}

\section{Conclusion}

The paper aims to evaluate the filterability of EPS solutions issued from $P$. cruentum cultures by modules equipped with organic membranes.

The establishment of the flux-TMP curves at various fluid velocities shows that the EPS solutions have a strong fouling power even at low EPS concentration. A critical flux for irreversibility $\mathrm{J}_{\mathrm{ci}}$ was found experimentally to be below $36 \mathrm{~L} \cdot \mathrm{m}^{-2} \cdot \mathrm{h}^{-1}$ and was estimated at about $32 \mathrm{~L} \cdot \mathrm{m}^{-2} \cdot \mathrm{h}^{-1}$ at a low EPS concentration which would correspond to a ratio $\mathrm{J}_{\mathrm{ci}} / \mathrm{J}_{\mathrm{lim}}$ close to 0.6 . The hydraulic resistance to the permeate flux is mainly irreversible at moderate pressure but the reversible fraction becomes predominant when approaching the limiting flux.

However, the permeate fluxes obtained at various fluid velocities allow estimating that a VRR of about 10 could be obtained by concentrating EPS solutions on organic membranes either in flat or tubular modules but not in a spiral-wound module. Working at the maximum temperature tolerated by the membrane (approximately $50{ }^{\circ} \mathrm{C}$ for PES membranes) should be considered, verifying that the polysaccharides will not be denatured for the intended use. 


\section{Acknowledgements}

This work was supported by the French Environment and Energy Management Agency (ADEME) under the Algoraff project, and by the Azm and Saade association (Tripoli, Lebanon).

We also thank the Algosolis platform (UMS CNRS 3722) for the supply of culture supernatants of Porphyridium cruentum, and Mr. Meignan (Ecolab, France) for graciously providing us with Ultrasil cleaning products and advice on their use.

\section{References}

[1] Arad, S. (Malis), Levy-Ontman, O., Red microalgal cell-wall polysaccharides:

biotechnological aspects. Curr. Opin. Biotechnol. $2010 ; 21,358-364$.

https://doi.org/10.1016/j.copbio.2010.02.008

[2] Pignolet, O., Jubeau, S., Vaca-Garcia, C., Michaud, P., Highly valuable microalgae: biochemical and topological aspects. J. Ind. Microbiol. Biotechnol.2013; 40, 781-796. https://doi.org/10.1007/s10295-013-1281-7

[3] Marcati, A., Ursu, A.V., Laroche, C., Soanen, N., Marchal, L., Jubeau, S., Djelveh, G., Michaud, P., Extraction and fractionation of polysaccharides and B-phycoerythrin from the microalga Porphyridium cruentum by membrane technology. Algal Res. 2014 ; 5, 258-263. https://doi.org/10.1016/j.algal.2014.03.006

[4] Delattre, C., Pierre, G., Laroche, C., Michaud, P., Production, extraction and characterization of microalgal and cyanobacterial exopolysaccharides. Biotechnol. Adv. 2016;34, 1159-1179. https://doi.org/10.1016/j.biotechadv.2016.08.001

[5] Ramus, J., The Production of Extracellular Polysaccharide by the Unicellular Red Alga Porphyridium Aerugineum. J. Phycol. 1972; 8, 97-111. https://doi.org/10.1111/j.1529-8817.1972.tb04007.x

[6] Adda, M., Merchuk, J.C., (Malis) Arad, S., Effect of nitrate on growth and production of cell-wall polysaccharide by the unicellular red alga Porphyridium. Biomass 1986; 10, 131-140. https://doi.org/10.1016/0144-4565(86)90061-2 
[7] Arad, S.M., Friedman, O.D., Rotem, A., Effect of Nitrogen on Polysaccharide Production in a Porphyridium sp. Appl. Environ. Microbiol. 1988; 54, 2411-2414.

[8] Csőgör, Z., Kiessling, B., Perner, I., Fleck, P., Posten, C., Growth and product formation of Porphyridium purpureum. J. Appl. Phycol. 2001; 13, 317-324. https://doi.org/10.1023/A:1017945513485

[9] Jubeau, S., Application du concept de bioraffinerie à la valorisation de la microalgue Porphyridium cruentum [Application of the biorefinery concept to the valorisation of the microalga Porphyridium cruentum] Ph.D., Nantes University; 2012.

[10] You, T., Barnett, S.M., Effect of light quality on production of extracellular polysaccharides and growth rate of Porphyridium cruentum. Biochem. Eng. J. 2004; 19, 251-258. https://doi.org/10.1016/j.bej.2004.02.004

[11] Geresh, S., Adin, I., Yarmolinsky, E., Karpasas, M., Characterization of the extracellular polysaccharide of Porphyridium sp: molecular weight determination and rheological properties. Carbohydr. Polym. 2002; 50, 183-189. https://doi.org/10.1016/S0144-8617(02)00019-X

[12] Eteshola, E., Karpasas, M., Arad, S., Gottlieb, M., Red microalga exopolysaccharides: 2 . Study of the rheology, morphology and thermal gelation of aqueous preparations. Acta Polym. 1998; 49, 549-556. https://doi.org/10.1002/(SICI)1521-4044(199810)49:10/11<549::AIDAPOL549>3.0.CO;2-T

[13] Patel, A.K., Laroche, C., Marcati, A., Ursu, A.V., Jubeau, S., Marchal, L., Petit, E., Djelyeh, G., Michaud, P., Separation and fractionation of exopolysaccharides from Porphyridium cruentum. Bioresour. Technol. 2013; 145, 345-350. https://doi.org/10.1016/j.biortech.2012.12.038

[14] Li, H., Li, Z., Xiong, S., Zhang, H., Li, N., Zhou, S., Liu, Y., Huang, Z., Pilot-scale isolation of bioactive extracellular polymeric substances from cell-free media of mass microalgal cultures using tangential-flow ultrafiltration. Process Biochem. 2011; 46, 1104-1109. https://doi.org/10.1016/j.procbio.2011.01.028 
[15] Morineau-Thomas, O., Jaouen, P., Legentilhomme, P., The role of exopolysaccharides in fouling phenomenon during ultrafiltration of microalgae (Chlorella sp. and Porphyridium purpureum): Advantage of a swirling decaying flow. Bioprocess Biosyst. Eng. 2002; 25, 35-42. https://doi.org/10.1007/s00449-001-0278-1

[16] Rossi, N., Derouiniot-Chaplain, M., Jaouen, P., Legentilhomme, P., Petit, I., Arthrospira platensis harvesting with membranes: Fouling phenomenon with limiting and critical flux. Bioresour. Technol.2008; 99, 6162-6167. https://doi.org/10.1016/j.biortech.2007.12.023

[17] Field, R.W., Wu, D., Howell, J.A., Gupta, B.B., Critical flux concept for microfiltration fouling. J. Membr. Sci. 1995; 100, 259-272. https://doi.org/10.1016/03767388(94)00265-Z

[18] Bacchin, P., Aimar, P., Sanchez, V., Model for colloidal fouling of membranes. AIChE J. 1995; 41, 368-376. https://doi.org/10.1002/aic.690410218

[19] Bacchin, P., Aimar, P., Field, R.W., Critical and sustainable fluxes: Theory, experiments and applications. J. Membr. Sci. 2006; 281, 42-69. https://doi.org/10.1016/j.memsci.2006.04.014

[20] DuBois, M., Gilles, K.A., Hamilton, J.K., Rebers, P.A., Smith, F., Colorimetric Method for Determination of Sugars and Related Substances. Anal. Chem. 1956; 28, 350-356. https://doi.org/10.1021/ac60111a017

[21] Michaels A.S., New separation technique for the process industries, Chem. Eng. Process.1968; 64, 31-43.

[22] Porter, M.C., Concentration Polarization with Membrane Ultrafiltration. Ind Eng Chem Prod Res Dev. 1972; 11, 234-248. https://doi.org/10.1021/i360043a002

[23] Espinasse, B., Bacchin, P., Aimar, P., On an experimental method to measure critical flux in ultrafiltration. Desalination 2002; 146, 91-96.

https://doi.org/10.1016/S0011-9164(02)00495-2 
[24] Espinasse, B., Bacchin, P., Aimar, P., Filtration method characterizing the reversibility of colloidal fouling layers at a membrane surface: analysis through critical flux and osmotic pressure. J. Colloid Interface Sci. 2008; 320, 483-490. https://doi.org/10.1016/j.jcis.2008.01.023

[25] Cheryan, M., Ultrafiltration and Microfiltration Handbook. CRC Press. 1998 [26] Rabiller-Baudry, M., Diagne, N.W., Lebordais, D., How the experimental knowledge of the irreversible fouling distribution can contribute to understand the fluid circulation in a spiral ultrafiltration membrane. Sep. Purif. Technol. 2014; 136, 157-167. https://doi.org/10.1016/j.seppur.2014.08.040

[27] Rezaei, H., Ashtiani, F.Z., Fouladitajar, A., Fouling behavior and performance of microfiltration membranes for whey treatment in steady and unsteady-state conditions. Braz. J. Chem. Eng. 2014; 31, 503-518. https://doi.org/10.1590/0104$6632.20140312 \mathrm{~s} 00002521$

[28] Ghosh, A.., Balakrishnan, M., Dua, M, Bhagat, J.., Ultrafiltration of sugarcane juice with spiral wound modules: on-site pilot trials. J. Membr. Sci. 2000; 174, 205-216. https://doi.org/10.1016/S0376-7388(00)00393-8

[29] Singh, V., Jain, P.K., Das, C., Performance of spiral wound ultrafiltration membrane module for with and without permeate recycle: Experimental and theoretical consideration. Desalination 2013; 322, 94-103. https://doi.org/10.1016/j.desal.2013.05.012 [30] Jönsson, A.-S,, Trägårdh, G., Fundamental principles of ultrafiltration. Chem. Eng. Process. Process Intensif. 1990; 27, 67-81. https://doi.org/10.1016/0255-2701(90)85011$\mathrm{R}$

[31] Blatt, W.F., Dravid, A., Michaels, A.S., Nelsen, L., Solute Polarization and Cake Formation in Membrane Ultrafiltration: Causes, Consequences, and Control Techniques, in: Flinn, J.E. (Ed.), Membrane Science and Technology: Industrial, Biological, and Waste Treatment Processes. Springer US, Boston, MA, 1970; 47-97. https://doi.org/10.1007/978-1-4684-1851-4_4 
[32] Gekas, V., Hallström, B., Mass transfer in the membrane concentration polarization layer under turbulent cross flow: I. Critical literature review and adaptation of existing sherwood correlations to membrane operations. J. Membr. Sci. 1987; 30, 153-170. https://doi.org/10.1016/S0376-7388(00)81349-6

[33] Balti, R., Le Balc'h, R., Brodu, N., Gilbert, M., Le Gouic, B., Le Gall, S., Sinquin, C., Massé, A., Concentration and purification of Porphyridium cruentum exopolysaccharides by membrane filtration at various cross-flow velocities. Process Biochem. 2018; In press, https://doi.org/10.1016/j.procbio.2018.06.021

[34] Campbell, M.J., Walter, R.P., McLoughlin, R., Knowles, C.J., Effect of temperature on protein conformation and activity during ultrafiltration. J. Membr. Sci.1993; 78, 3543. https://doi.org/10.1016/0376-7388(93)85245-R

[35] Wu, D., Howell, J.., Field, R.., Critical flux measurement for model colloids. J. Membr. Sci. 1999; 152, 89-98. https://doi.org/10.1016/S0376-7388(98)00200-2 [36] Cho, B.., Fane, A.., Fouling transients in nominally sub-critical flux operation of a membrane bioreactor. J. Membr. Sci. 2002; 209, 391-403. https://doi.org/10.1016/S03767388(02)00321-6 


\section{Appendix 1 - Relation between the limiting flux $J_{\lim }$ and the tangential velocity $V$}

\section{for a set of filtrations carried out on the same solution and at the same temperature}

The film theory leads to the following relation as soon as the concentration polarization is not negligible (see for instance Jönsson and Trägårdh [30]):

$$
J=k \ln \frac{C_{w}-C_{p}}{C_{0}-C_{p}}
$$

where $\mathrm{J}$ is the permeate flux, $\mathrm{k}$ the solute mass transfer coefficient of the solute in the concentration boundary layer and $\mathrm{C}_{0}, \mathrm{C}_{\mathrm{w}}$ and $\mathrm{C}_{\mathrm{p}}$ are the concentration in solute in the bulk, at the membrane wall and in the permeate respectively. At the limiting flux and in the presence of a solute gel at the membrane wall, Equation A1.1 is reduced to:

$$
J_{\text {lim }}=k \ln \frac{C_{g}}{C_{0}}
$$

with $\mathrm{C}_{\mathrm{g}}$, the gel concentration of the solute, which can be often considered as independent of the flow pattern as a first approximation (more precisely, the gel layer model supposes the existence of a fairly well-defined gel layer for a concentration in solutes independent of the operating parameters and the flow pattern, so long as the solutes retention remain constant [25, section 4G]. Otherwise, the mass transfer coefficient $\mathrm{k}$ can be estimated by correlations having the following form:

$$
S h=A R e^{a} S c^{b}
$$

where Sh, Re and Sc are the Sherwood, Reynolds and Schmidt numbers, and A, a and $b$ constants that on the state development of the fluid velocity and the solute concentration along the channel. For turbulent flow, $a$ is known to be comprised between 0.75 and 0.9 [32]. If all the experiments are carried out with the same solution and at the same temperature, all variables in equation (A.1.3) are constants except the tangential velocity $\mathrm{V}$, and the mass transfer coefficient $\mathrm{k}$ and (A.1.2) and (A.1.3) reduce respectively to

$$
k=B \times V^{a}
$$

and: 


$$
J_{\text {lim }}=C \times V^{a}
$$

where $\mathrm{B}$ and $\mathrm{C}$ are two constants.

\section{Appendix 2 - Comparison of wall shear stress for typical hydrodynamic conditions in spiral wound (SW), plate and frame (PF) and tubular (TUB) modules}

The analysis relies on a simplified representation of the fluid flow in the module that assumes in particular (i) a homogeneous distribution of the feed over the width of the membrane, (ii) an exclusively axial fluid flow and (iii) a predominant effect of the frictional losses along the membrane in front of those at the inlet and the outlet of the module. These assumptions are obviously questionable, in particular for spiral and plate and frame modules.

Wall shear stress $\tau_{\mathrm{w}}$ along the membrane were estimated through the following equation valid in turbulent flow for a free fluid vein:

$$
\tau_{W}=\frac{1}{2} \rho V^{2} f
$$

with $f=\frac{0.079}{R e^{025}}$ (Blasius law for a conduct with a hydraulically smooth surface)

$$
R e=\frac{D_{h} V \rho}{\eta}
$$

where $f$ is the friction factor, Re the Reynolds number, $V$ the (mean) fluid velocity, $D_{h}$ the hydraulic diameter of the flow channel $\left(D_{\mathrm{h}}=\mathrm{D}\right.$ for a cylindrical tube of diameter $\mathrm{D}$ and $D_{\mathrm{h}} \approx 2$ e for a flat, large, rectangular channel height e), $\rho$ and $\eta$ the density and dynamic viscosity of the fluid which were assimilated here to those of pure water at 20 ${ }^{\circ} \mathrm{C}$ (i.e. $\rho=10^{3} \mathrm{~kg} \cdot \mathrm{m}^{-3}$ and $\rho / \eta=10^{6} \mathrm{~m}^{2} \cdot \mathrm{s}^{-1}$ ). For SW and PF modules, computations 
were performed on the basis of the hydraulic analogy between exp. $1^{*}$ and 1 (same curve flux-TMP) as explained in Table A.2.1.

Tubular module. As all friction is exerted on the membrane (the whole volume inside the tube is occupied by the fluid), $\tau_{\mathrm{w}}$ can be computed directly through equation (A2.1).

Spiral-wound module. Shear stress have been estimated based on the hydraulic similitude between runs 1 and $1^{*}$ whose flux-TMP curves are superimposable. The similitude allows hypothesizing that the mean wall shear stresses are quite the same in the two experiments. Therefore, the mean shear stress $\tau_{\mathrm{w}, \mathrm{SW}}$ for run $1\left(\mathrm{~V}=0.3 \mathrm{~m} . \mathrm{s}^{-1}, \mathrm{e}=1.5 \mathrm{~mm}\right.$ with a 46 mil spacer) was estimated in the hydraulic conditions of run $1 *\left(\nabla^{*}=1.0 \mathrm{~m} . \mathrm{s}^{-1}\right.$ for $\mathrm{e}=0.5 \mathrm{~mm}$ with no spacer).

The shear stress $\tau_{\mathrm{w}, \mathrm{PF}}$ on a flat membrane in a FP module was computed assuming that the hydraulic analogy between runs 1 and $1^{*}$ is kept at higher velocities, i.e assuming that $\tau_{\mathrm{w}, \mathrm{PF}}\left(\mathrm{V}=1.0 \mathrm{~m} . \mathrm{s}^{-1}, \mathrm{e}=1.5 \mathrm{~mm}\right.$ with a 46 mil spacer $)$ is close to the shear stress in a free vein fluid, $0.5 \mathrm{~mm}$ height at a fluid velocity $\mathrm{V}=1.0 \times(1.0 / 0.3)=3.33 \mathrm{~m} . \mathrm{s}^{-1}$.

The calculations lead to the following values of wall shear stresses: $\tau_{\mathrm{w}, \mathrm{TUB}}=86$ $\mathrm{Pa}, \tau_{\mathrm{w}, \mathrm{SW}}=8 \mathrm{~Pa}$ and $\tau_{\mathrm{w}, \mathrm{PF}}=58 \mathrm{~Pa}$.

NB: the flow is laminar for the equivalent hydrodynamic conditions for the SW module $(\operatorname{Re}=1000)$, but not too far from the turbulent flow so that the friction factor $f$ computed from the Blasius law and from the law valid in laminar flow, $\mathrm{f}=16 / \mathrm{Re}$ are close (resp. 0.014 and 0.016 ).

\section{Insert Table A.2.1. here}


Figure 1. Schematic diagram of the cross-flow filtration set-up. 1: feeding tank, 2: hotplate stirrer, 3: cryothermostat, 4: feed pump, 5: pulse dampener, 6: membrane module, 7: measuring cylinder (permeate recovery), 8: precision balance, 9: pressure gauge at the module inlet, 10: feed input pressure transmitter, 11: pressure gauge at the module outlet, 12: retentate output pressure transmitter, 13: throttling valve (back pressure), 14: permeate temperature sensor (thermocouple), 15: retentate temperature sensor (thermocouple), 16: data acquisition system, 17: PC-data logging system, 18: air purge valve, 19: safety valve, 20: drain valve.

Figure 2. Steady state permeation flux J against TMP at various cross-flow velocities. Experimental conditions: exp. No. 1-4, membrane sample $\mathrm{MQ}_{2}, \mathrm{e}=1.5 \mathrm{~mm}$ with feed spacer, VRR $=1$, EPS solution $\mathrm{S}_{\AA}$ $\left(\mathrm{c}_{0}=0.10 \mathrm{~kg} \mathrm{GlcEq} \cdot \mathrm{m}^{-3}\right), \mathrm{T}=20^{\circ} \mathrm{C}, \mathrm{V}=0.3,0.6,1.0$ and $1.2 \mathrm{~m} \cdot \mathrm{s}^{-1}$. Limiting permeate fluxes for the various velocities are indicated on the diagram.

Figure 3. Limiting permeate flux $\mathrm{J}_{\lim }$ against the cross-flow velocity $\mathrm{V}$ on linear (a) and logarithmic scales (b). Experimental conditions: exp. No. 1-4, membrane sample $\mathrm{MQ}_{2}, \mathrm{e}=1.5 \mathrm{~mm}$ with feed spacer, $\mathrm{VRR}=$ 1 , EPS solution $\mathrm{S}_{\mathrm{A}}\left(\mathrm{c}_{0}=0.10 \mathrm{~kg}\right.$ GlcEq. $\left.\mathrm{m}^{-3}\right), \mathrm{T}=20^{\circ} \mathrm{C}$.

Figure 4. Evolution of permeate flux versus time during cell-free medium filtration, with a step-by-step increase TMP (a) or at the nominal TMP ${ }_{n}$ of 0.57 bar (b). Experimental conditions. exp. no 3 (b) and 5 (a), membrane sample $\mathrm{MQ}_{2}, \mathrm{e}=1.5 \mathrm{~mm}$ with feed spacer, $\mathrm{VRR}=1$, EPS solution $\mathrm{S}_{\mathrm{A}}\left(\mathrm{c}_{0}=0.10 \mathrm{~kg} \mathrm{GlcEq} \cdot \mathrm{m}^{-3}\right)$, $\mathrm{T}=20^{\circ} \mathrm{C}, \mathrm{V}=1.0 \mathrm{~m} \cdot \mathrm{s}^{-1}$.

Figure 5. Effect of EPS concentration $\mathrm{c}_{0}$ on the steady-state permeation flux at various TMP. Experimental conditions: exp. No. 1, 6, 7, 9, membrane sample $\mathrm{MQ}_{2}$, e $=1.5 \mathrm{~mm}$ with feed spacer, VRR $=1$, EPS solution $\mathrm{S}_{\mathrm{A}}\left(0.10 \mathrm{~kg}\right.$ GlcEq. $\left.\mathrm{m}^{-3}\right), \mathrm{S}_{\mathrm{B}}\left(0.16 \mathrm{~kg}\right.$ GlcEq.m $\left.{ }^{-3}\right), \mathrm{S}_{\mathrm{Bcd} 1}\left(0.64 \mathrm{~kg}\right.$ GlcEq. $\left.\mathrm{m}^{-3}\right)$ and $\mathrm{S}_{\mathrm{Bcd} 2}(1.06 \mathrm{~kg}$ GlcEq. $\left.\mathrm{m}^{-3}\right), \mathrm{T}=20^{\circ} \mathrm{C}, \mathrm{V}=1.0 \mathrm{~m} \cdot \mathrm{s}^{-1}$. Limiting permeate fluxes values for the various EPS concentrations are indicated on the diagram.

Figure 6. Curve $\log (\mathrm{J})$ versus $\log \left(\mathrm{c}_{0}\right)$, with $\mathrm{J}$ the limiting permeate fluxes and $\mathrm{c}_{0}$ the EPS concentration. Experimental conditions: exp. No. 3, 6, 7, 9, membrane sample $\mathrm{MQ}_{2}, \mathrm{e}=1.5 \mathrm{~mm}$ with feed spacer, $\mathrm{VRR}=$ 1 , EPS solution $\mathrm{S}_{\mathrm{A}}\left(0.10 \mathrm{~kg} \mathrm{GlcEq} \cdot \mathrm{m}^{-3}\right), \mathrm{S}_{\mathrm{B}}\left(0.16 \mathrm{~kg} \mathrm{GlcEq} \cdot \mathrm{m}^{-3}\right), \mathrm{S}_{\mathrm{Bcd} 1}\left(0.64 \mathrm{~kg} \mathrm{GlcEq} \cdot \mathrm{m}^{-3}\right)$ and $\mathrm{S}_{\mathrm{Bcd} 2}(1.06$ kg GlcEq. $\left.\mathrm{m}^{-3}\right), \mathrm{T}=20^{\circ} \mathrm{C}, \mathrm{V}=1.0 \mathrm{~m} \cdot \mathrm{s}^{-1}$.

Figure 7. Effect of temperature on steady-state permeation flux $\mathrm{J}$ at various TMP. Experimental conditions: exp. No. 10-11, membrane sample $M Q_{3}, \mathrm{e}=1.5 \mathrm{~mm}$ with feed spacer, $\mathrm{VRR}=1$, EPS solution $\mathrm{S}_{\mathrm{Bd} 1}(0.64 \mathrm{k}$ GlcEq. $\mathrm{m}^{-3}, \mathrm{~T}=20$ and $40^{\circ} \mathrm{C}, \mathrm{Y}=1.0 \mathrm{~m} \cdot \mathrm{s}^{-1}$. Limiting permeate fluxes values for the various temperatures are indicated on the diagram.

Figure 8. Evolution of hydraulic resistances $\left(\mathrm{R}_{\mathrm{m}}, \mathrm{R}_{\mathrm{rev}}\right.$ and $\left.\mathrm{R}_{\text {irrev }}\right)$. Exp. 1, 2, 3, 4 (influence of cross-flow velocity $\mathrm{V}$ on EPS filterability), exp. 5 (UF at nominal TMP for $\mathrm{V}=1.0 \mathrm{~m} \cdot \mathrm{s}^{-1}$ ), exp. 6,7 \& 8, 9 (influence of EPS concentration $\mathbf{c}_{0}$, exp. $10 \& 11$ (evaluation of the critical flux), exp. $12 \& 13$ (influence of temperature). Operating conditions are mentioned above the results of each experiment.

Figure 9. Evaluation of the critical flux for irreversibility $\mathrm{J}_{\mathrm{ci}}$ by the method of square wave of applied pressure, with step amplitude of 0.20 bar (a, exp. No. 10) or 0.10 bar (b, exp. No. 11). Experimental conditions: membrane sample $\mathrm{MQ}_{2}, \mathrm{e}=1.5 \mathrm{~mm}$ with feed spacer, $\mathrm{VRR}=1, \mathrm{EPS}$ solution $\mathrm{S}_{\mathrm{B}}(0.16 \mathrm{~kg}$ GIcEq. $\left.\mathrm{m}^{-3}\right), \mathrm{T}=20^{\circ} \mathrm{C}, \mathrm{V}=1.0 \mathrm{~m} \cdot \mathrm{s}^{-1}$.

Figure 10. Evolution of the irreversible fouling resistance $\mathrm{R}_{\text {irrev }}^{*}$ against the transmembrane pressure with the technique of the square wave of applied pressure. Exp. No. 10 (square marks) and 11 (round marks).

Same conditions as for Figure 9. 


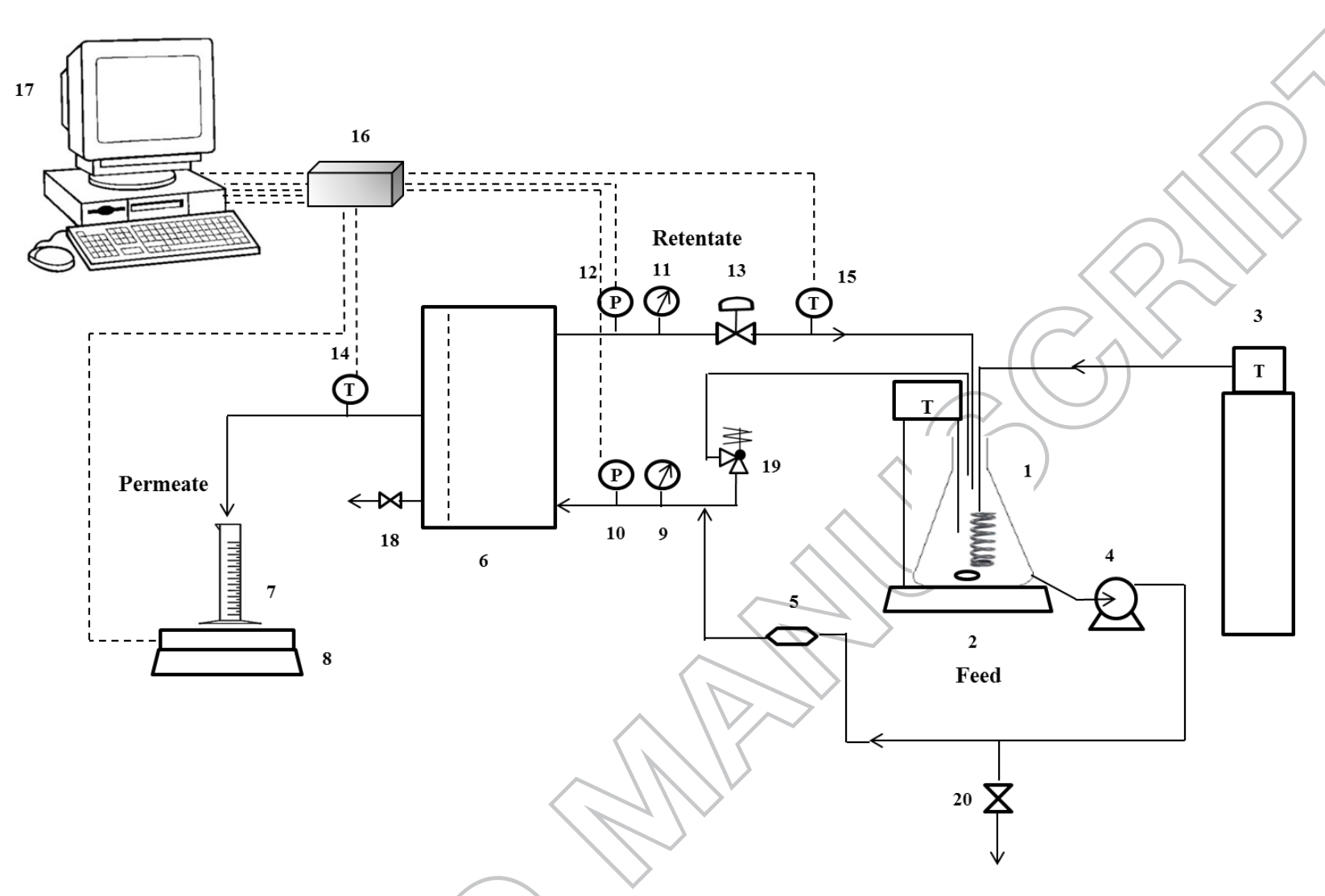




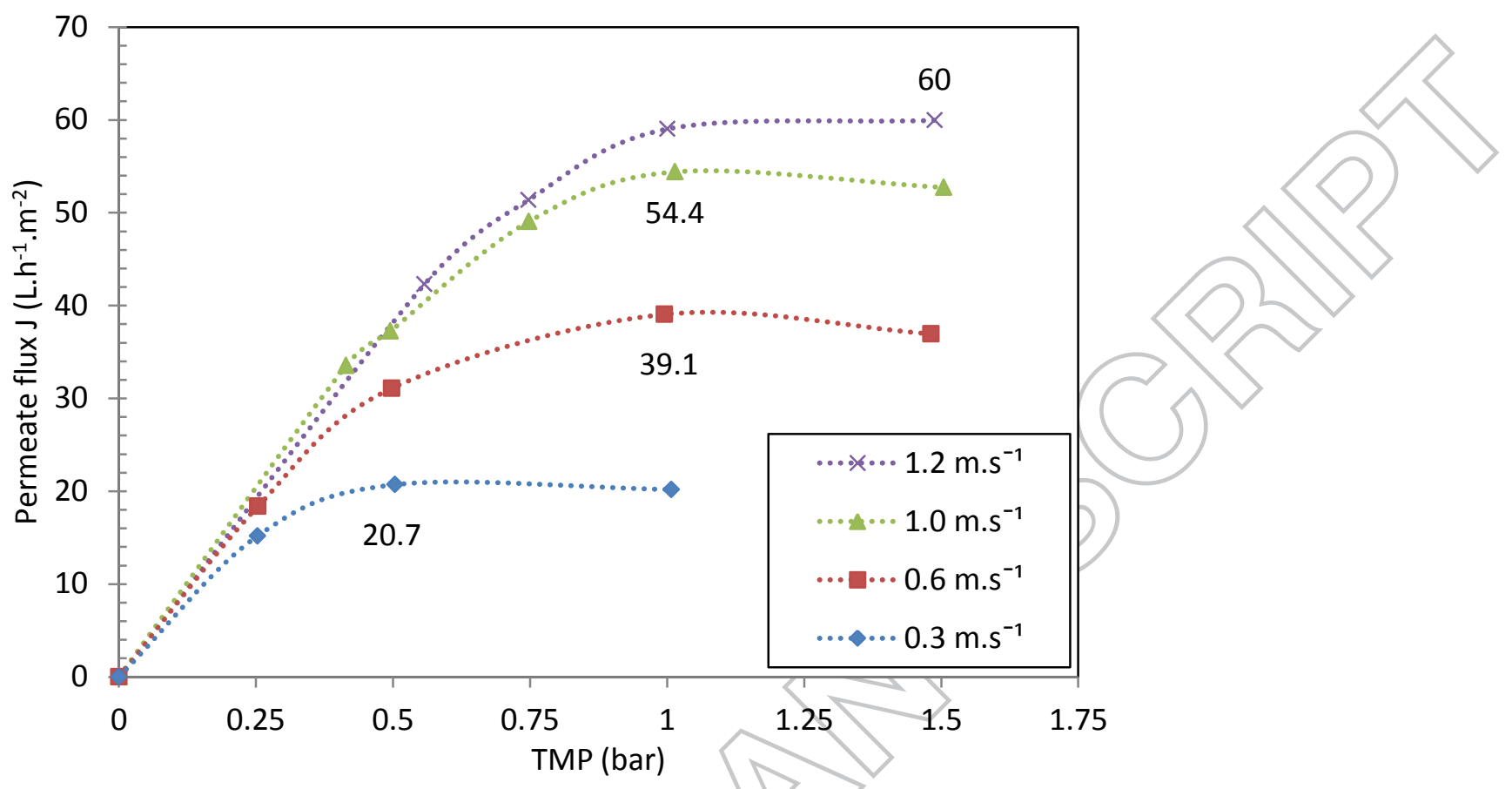


a)

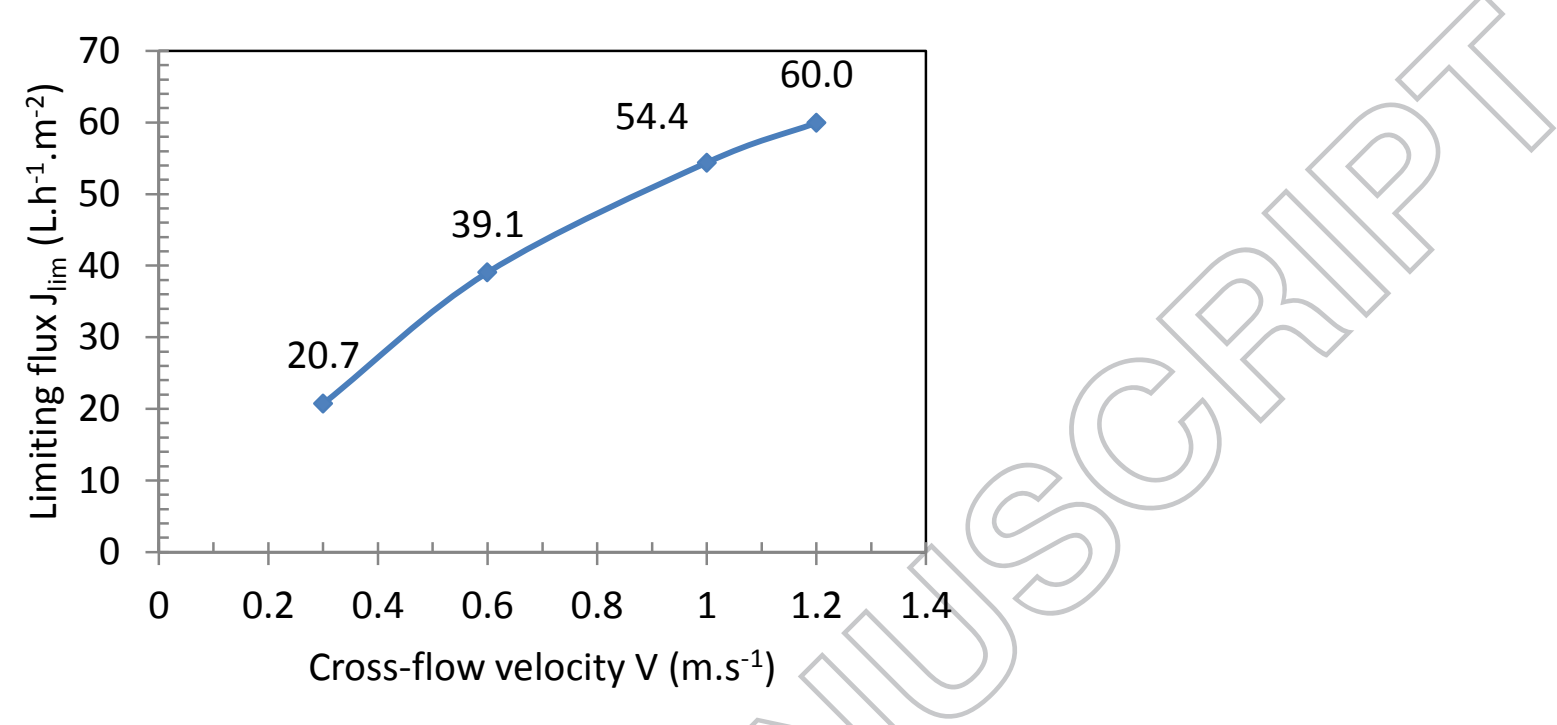

b)

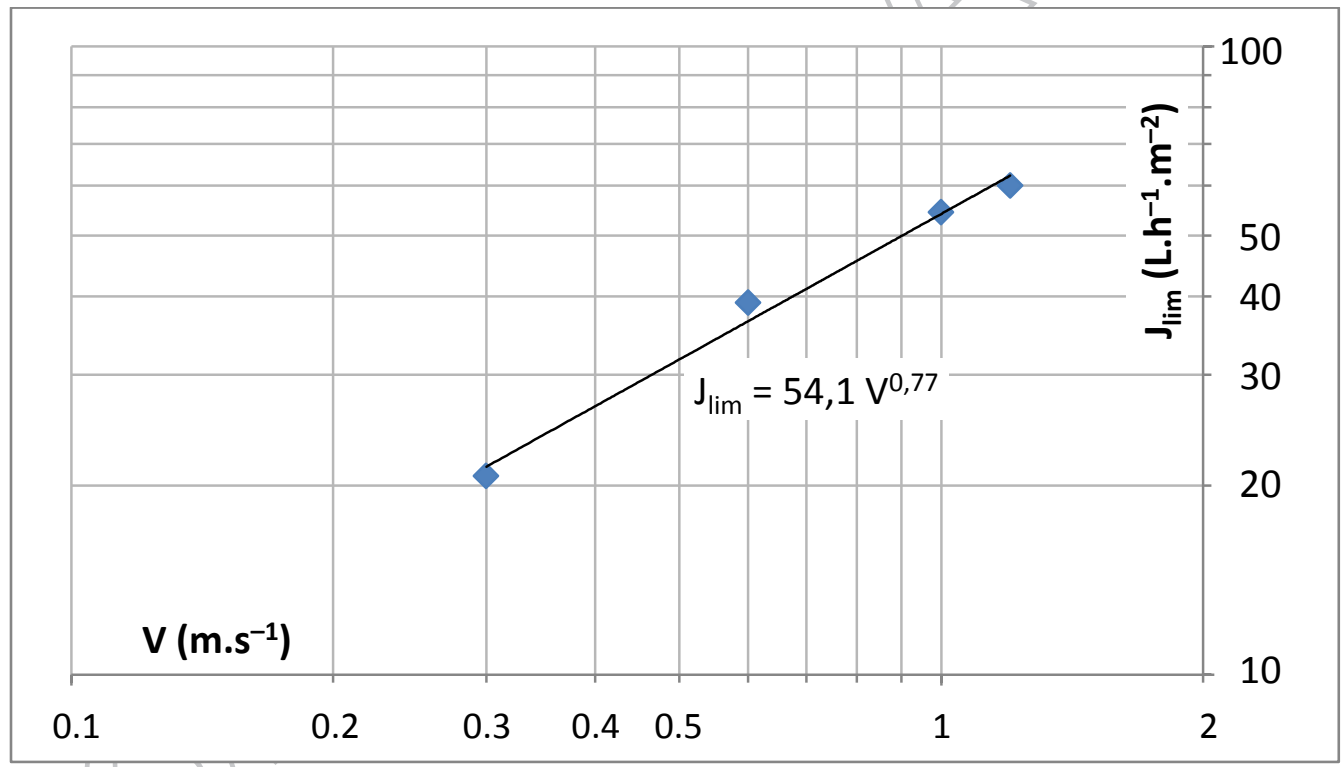


a)

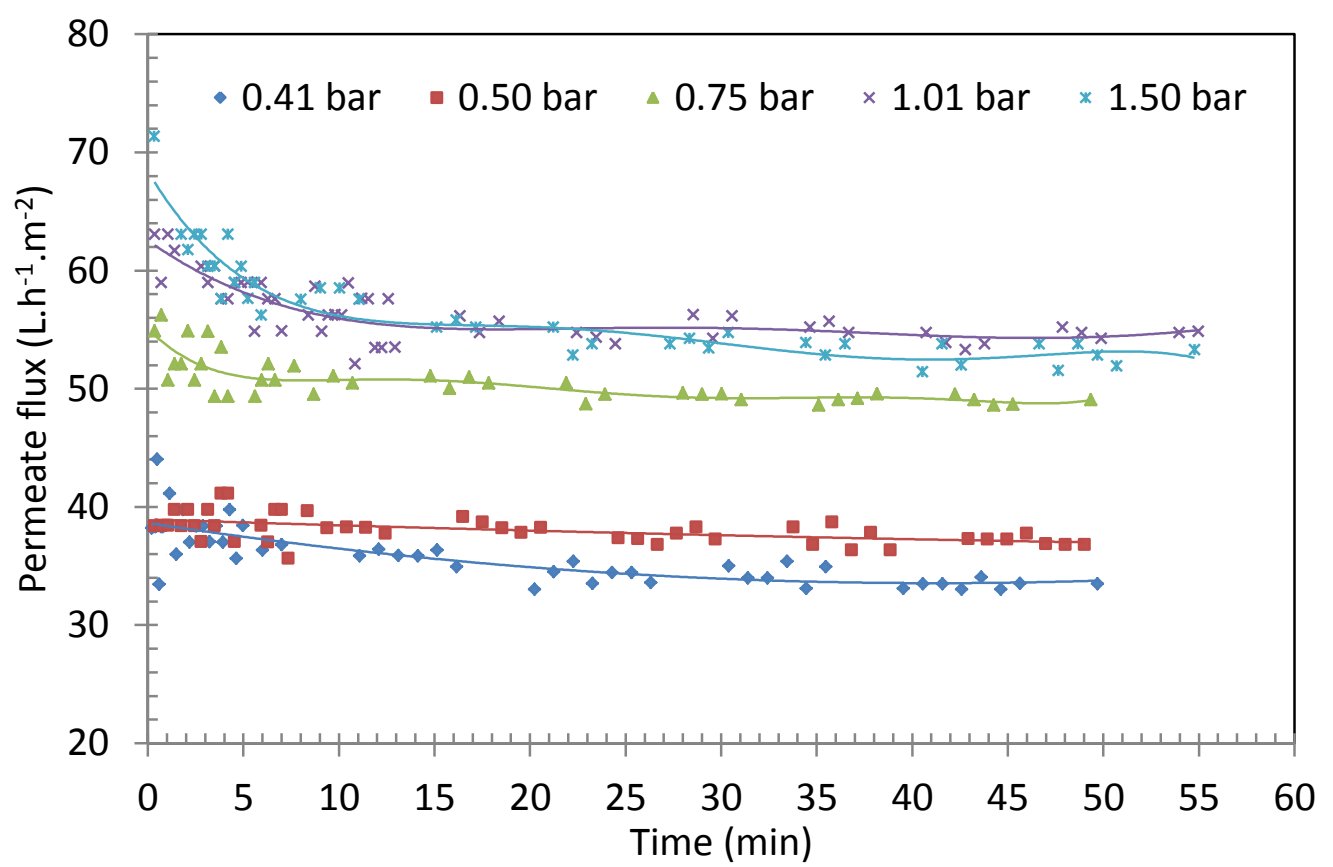

b)

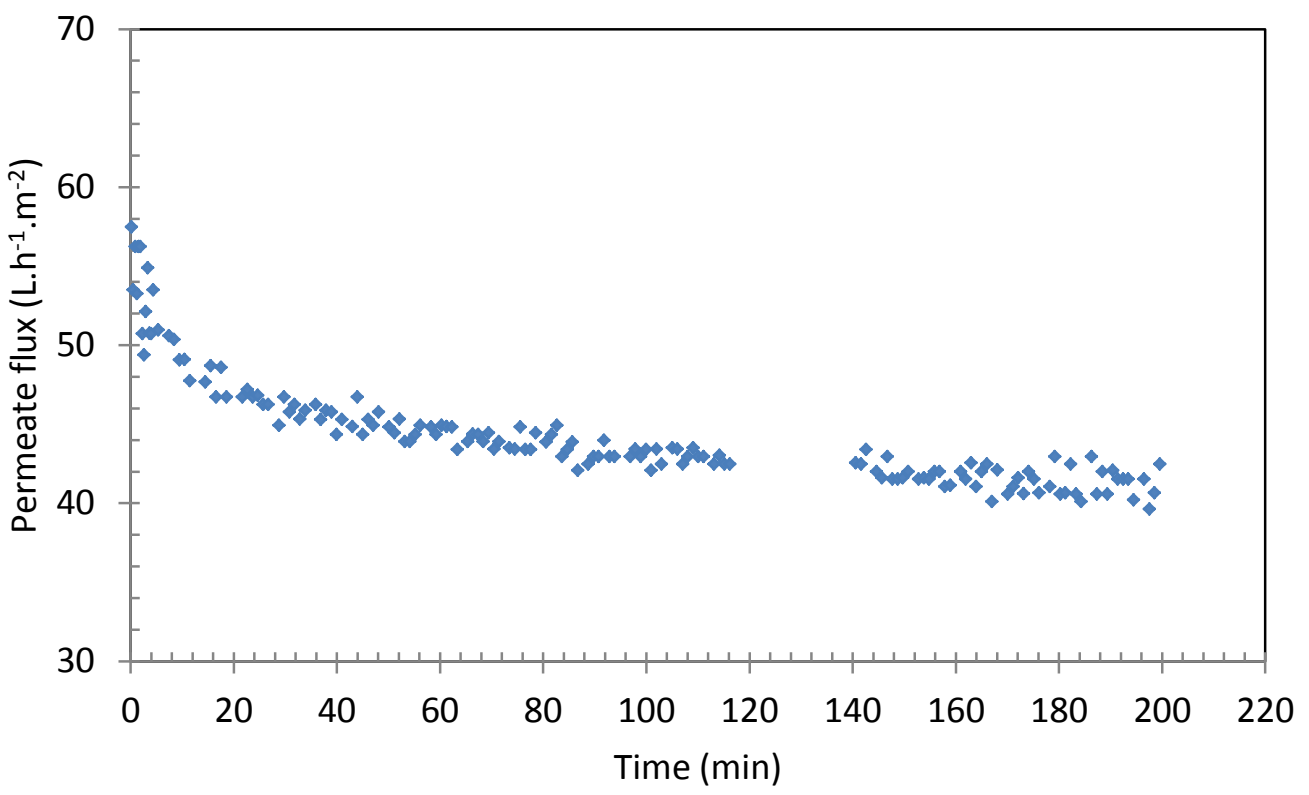



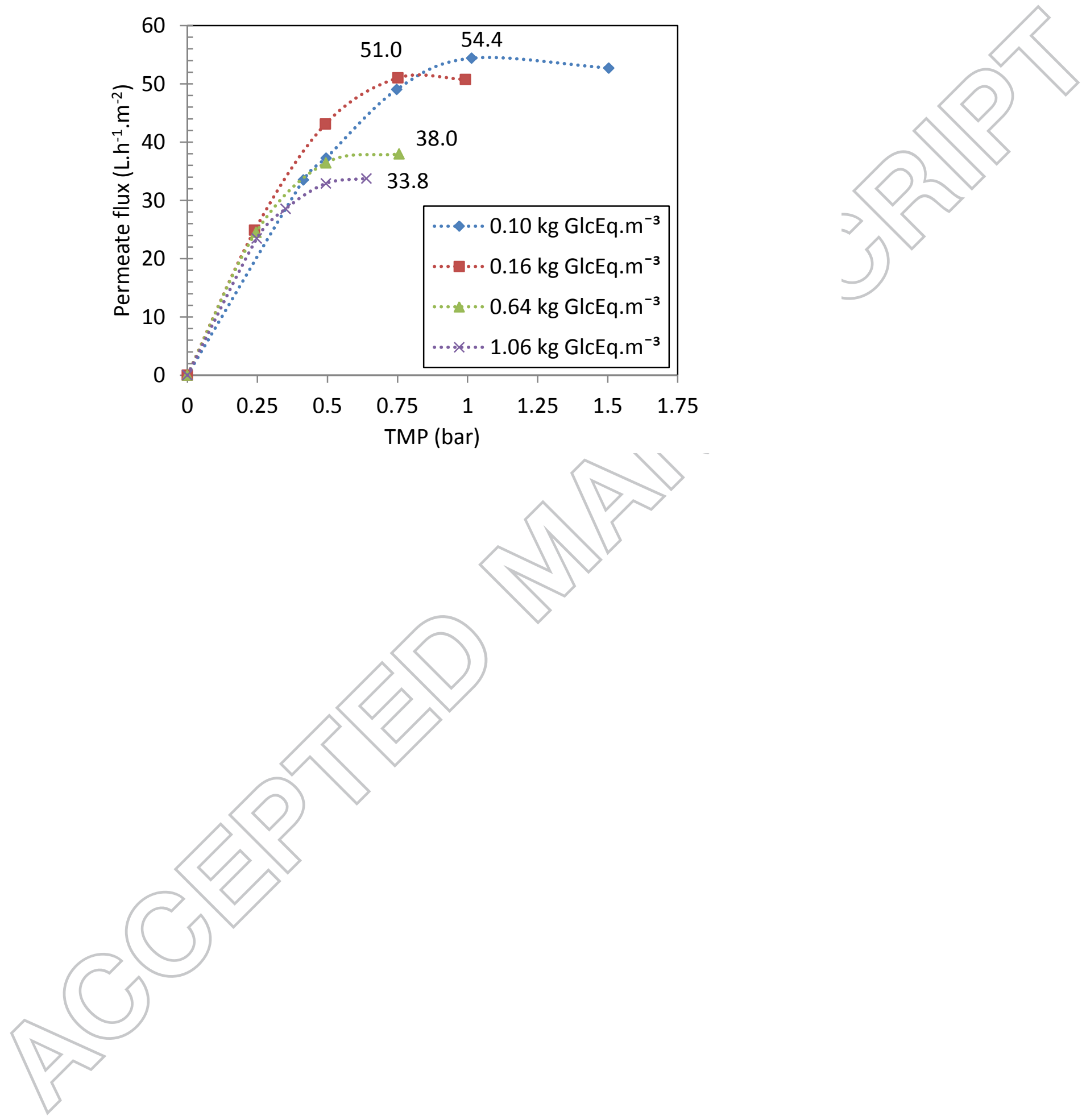


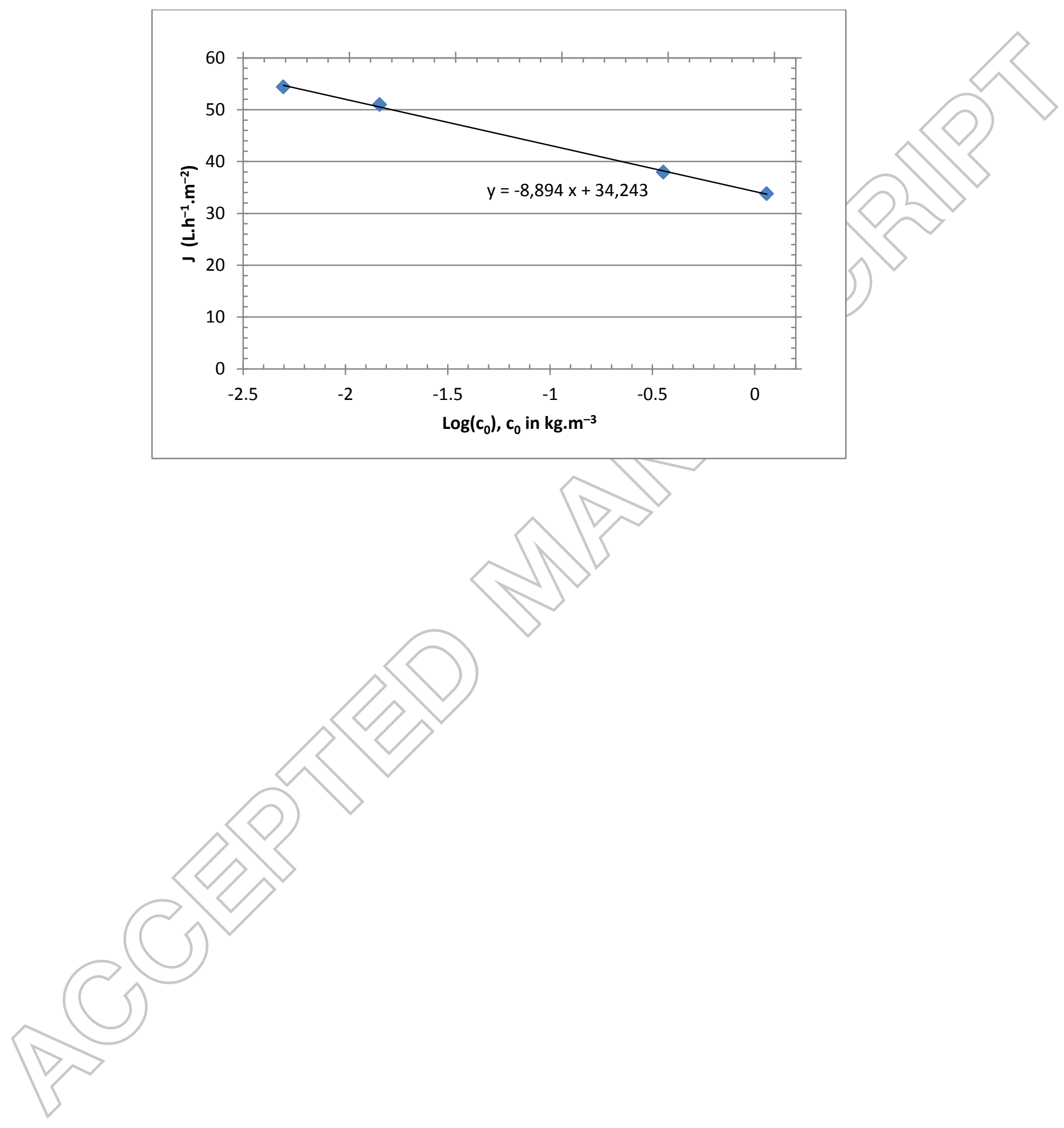




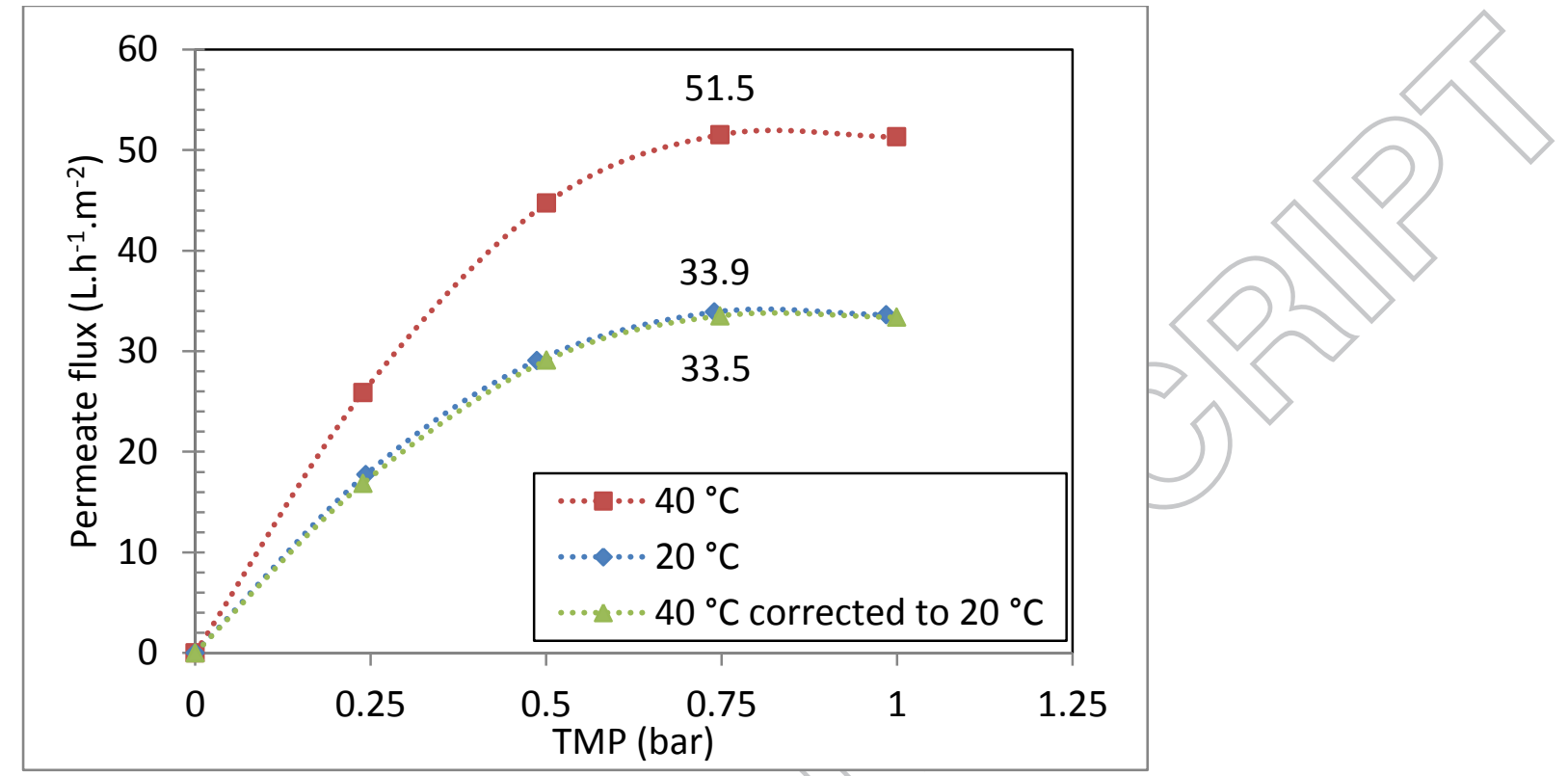




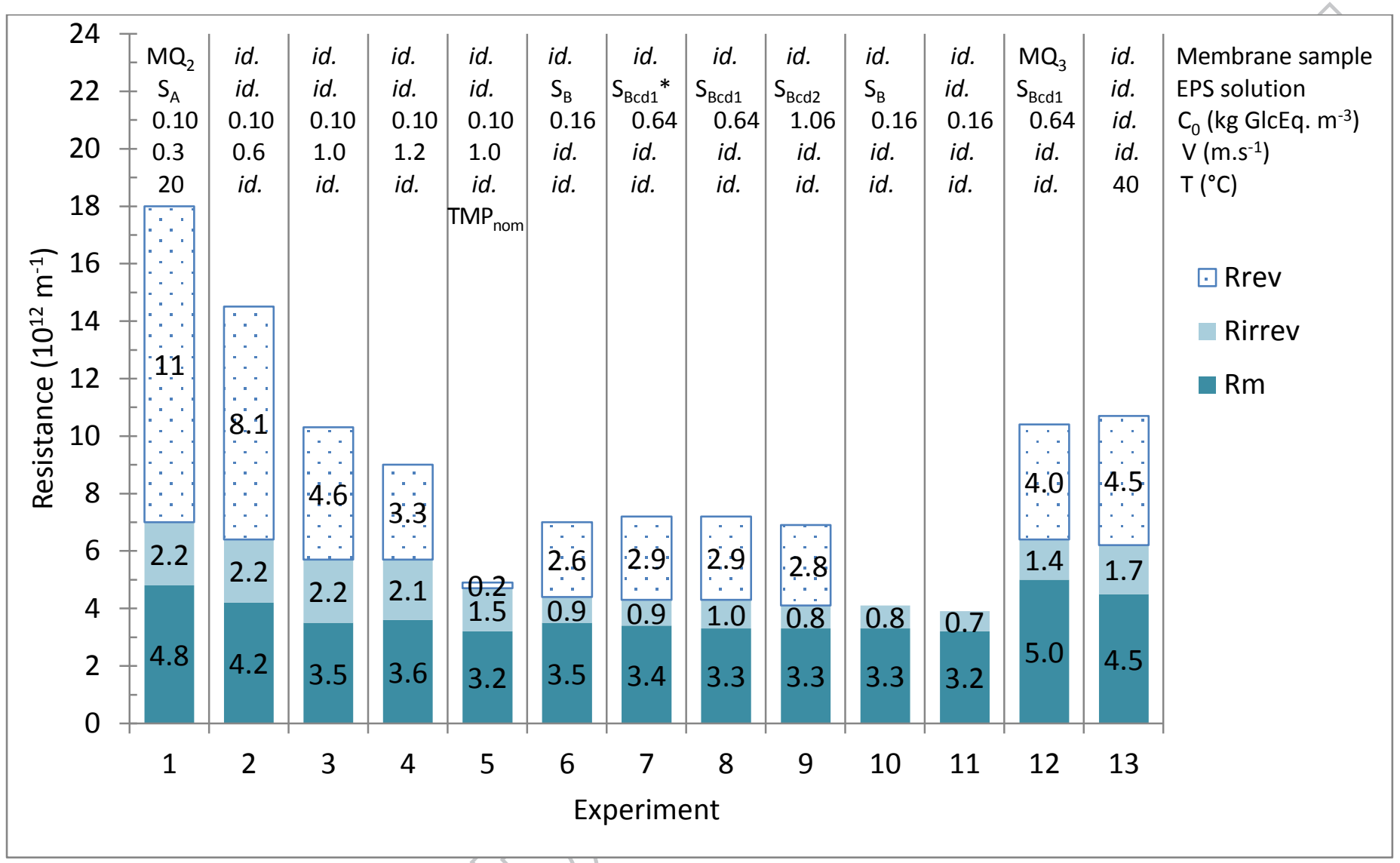


a)

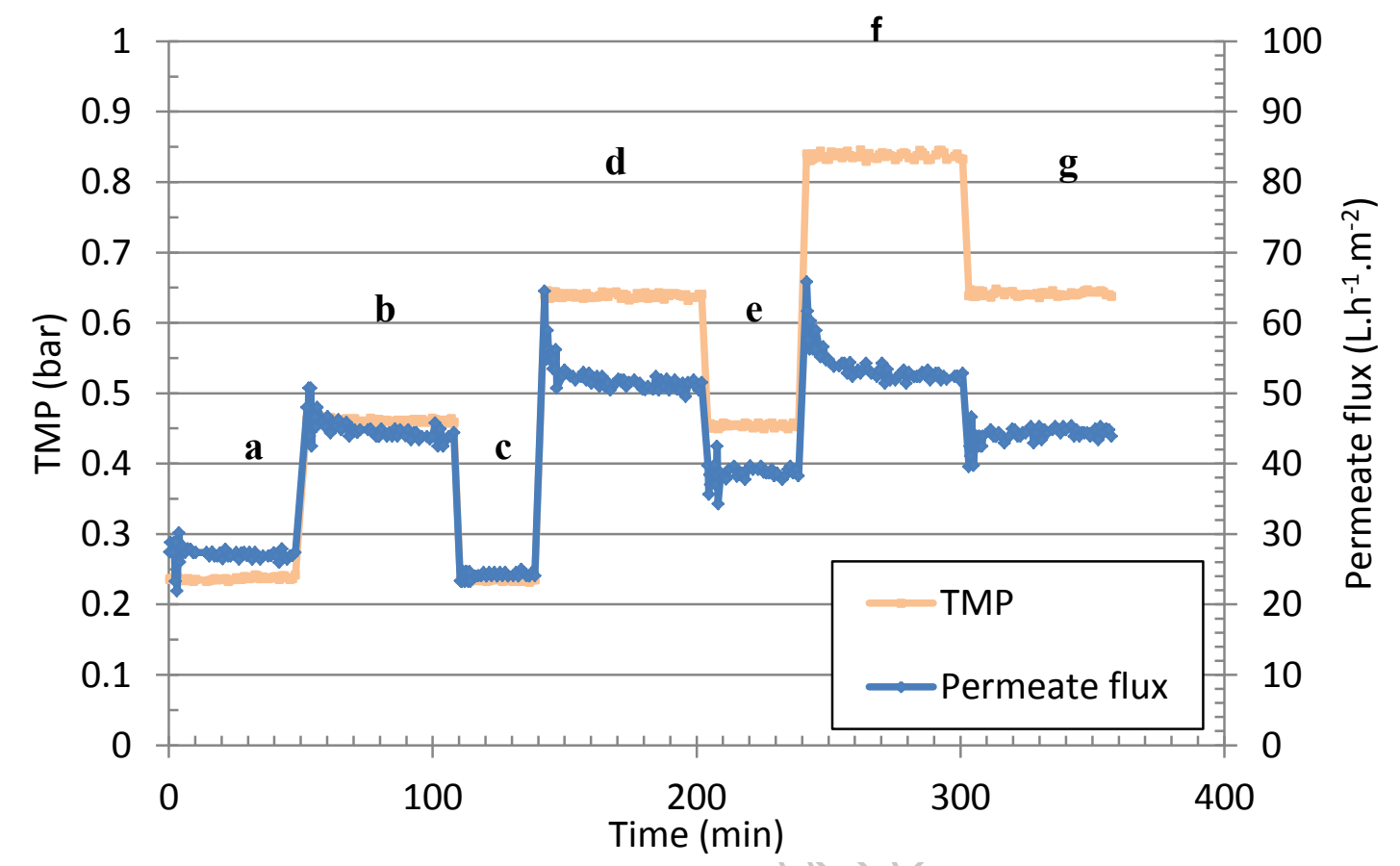

b)

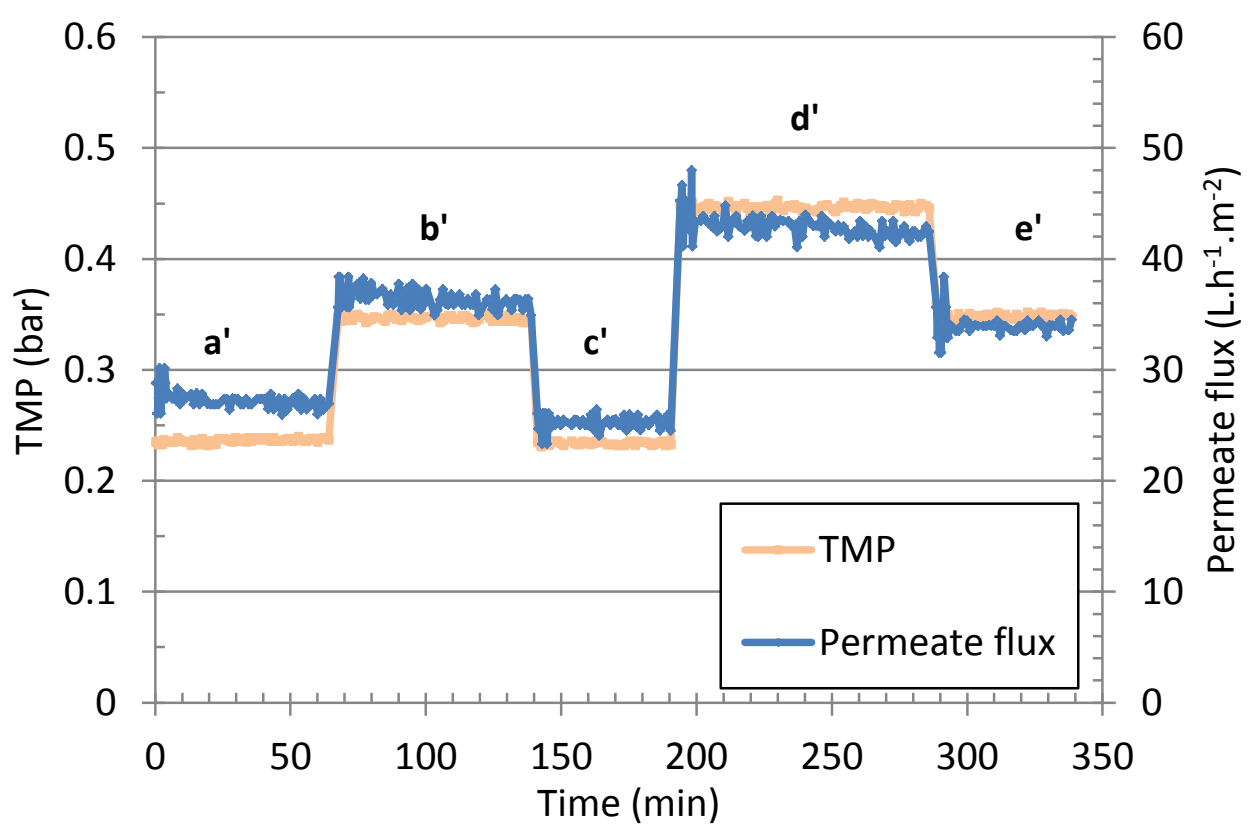




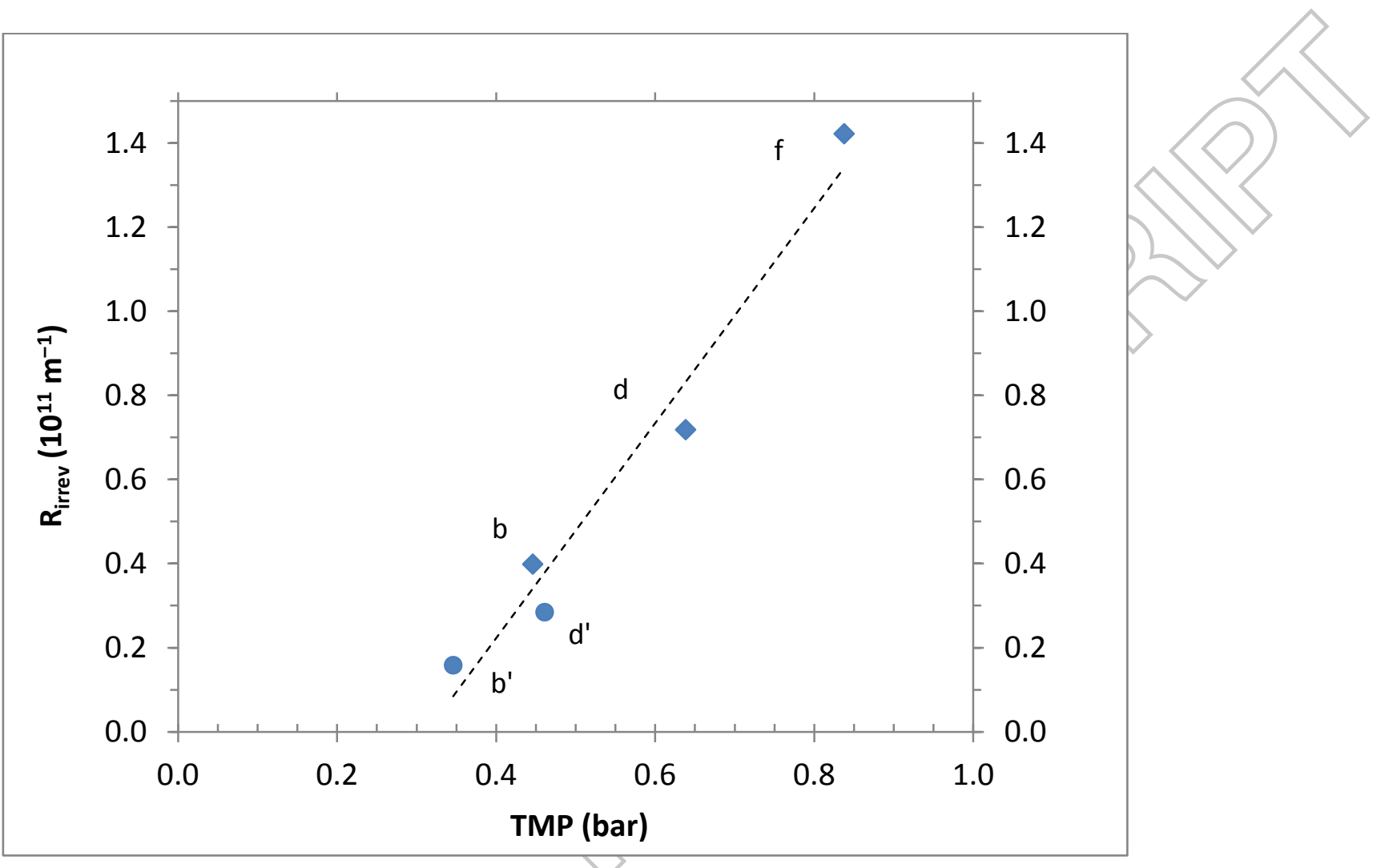


Table 1. Composition of the modified BBM cuture medium.

Table 2. Characteristics of the EPS solutions filtered

Table 3. Experiments carried out and their operating conditions.

Table 4. Limiting permeate fluxes and TMP obtained during EPS fouling experiments at $20{ }^{\circ} \mathrm{C}$, with the membrane sample $\mathrm{MQ}_{2}$. Experimental conditions: exp. No. 1-4, e $=1.5$ $\mathrm{mm}$ with feed spacer, VRR $=1$, EPS solution $\mathrm{S}_{\mathrm{A}}\left(\mathrm{c}_{0}=0.10 \mathrm{~kg} \mathrm{GlcEq} \cdot \mathrm{m}^{-3}\right), \mathrm{T}=20^{\circ} \mathrm{C}, \mathrm{V}$ $=1.0 \mathrm{~m} \cdot \mathrm{s}^{-1}$.

Table 5. Comparison of filtration performance of EPS solution and skim milk at close temperatures

Table A.2.1. Hydrodynamic conditions considered in spiral-wound, plate and frame and tubular modules; column a) real conditions in the module, column b) equivalent conditions considered in this work for the estimation of wall shear stress. Fluid properties assimilated to those of pure water at $20^{\circ} \mathrm{C}$ in all cases. 
Table 3. Composition of the modified BBM cuture medium.

\begin{tabular}{|c|c|c|c|}
\hline \multicolumn{4}{|c|}{ Composition of modified BBM medium $\left(\mathrm{kg} \cdot \mathrm{m}^{-3}\right)$} \\
\hline $\mathrm{NaNO}_{3}$ & 1.5 & & \\
\hline $\mathrm{MgSO}_{4}, 7 \mathrm{H}_{2} \mathrm{O}$ & 0.225 & \multicolumn{2}{|c|}{ Composition of additive 1} \\
\hline $\mathrm{CaCl}_{2}, 2 \mathrm{H}_{2} \mathrm{O}$ & 0.025 & $\mathrm{ZnSO}_{4}, 7 \mathrm{H}_{2} \mathrm{O}$ & 0.2 \\
\hline $\mathrm{Na}_{2}$ EDTA, $2 \mathrm{H}_{2} \mathrm{O}$ & 0.05 & \multicolumn{2}{|c|}{$\mathrm{Co}\left(\mathrm{NO}_{3}\right)_{2}, 6 \mathrm{H}_{2} \mathrm{O} \bigcirc 0.044$} \\
\hline $\mathrm{FeSO}_{4}, 7 \mathrm{H}_{2} \mathrm{O}$ & 0.014 & \multicolumn{2}{|c|}{$\left.\mathrm{CuSO}_{4}, 5 \mathrm{H}_{2} \mathrm{O}\right) \quad 0.1$} \\
\hline $\mathrm{K}_{2} \mathrm{HPO}_{4}$ & 0.15 & \multicolumn{2}{|c|}{$\mathrm{H}_{3} \mathrm{BO}_{3}>2.9$} \\
\hline $\mathrm{KH}_{2} \mathrm{PO}_{4}$ & 0.123 & \multicolumn{2}{|c|}{$\mathrm{MnCl}_{2}, 4 \mathrm{H}_{2} \mathrm{O}$} \\
\hline $\mathrm{NaCl}$ & 15 & & \\
\hline $\mathrm{NaHCO}_{3}$ & 1.26 & \multicolumn{2}{|c|}{ Composition of additive 2} \\
\hline \multicolumn{2}{|c|}{$+1 \mathrm{~L}^{-\mathrm{m}^{-3}}$ of additive 1 and $1 \mathrm{~L}^{-\mathrm{m}^{-3}}$ of additive 2} & $\mathrm{MoO}_{3}$ & 0.2 \\
\hline
\end{tabular}


Table 4. Characteristics of the EPS solutions filtered.

\begin{tabular}{|c|c|c|c|c|}
\hline Solution & $\mathrm{S}_{\mathrm{A}}$ & $\mathrm{S}_{\mathrm{B}}$ & $\mathrm{S}_{\mathrm{Bcd} 1 \&} \mathrm{~S}_{\mathrm{Bcd} 1 *}$ & $\mathrm{~S}_{\mathrm{Bcd} 2}$ \\
\hline EPS concentration (kg GlcEq.m ${ }^{-3}$ ) & 0.10 & 0.16 & 0.64 & \\
\hline Protein concentration ( $\mathrm{kg} \mathrm{BSAEq} \cdot \mathrm{m}^{-3}$ ) & 0.0097 & 0.014 & 0.021 & \\
\hline Protein to EPS ratio (w/w\%) & 9.7 & 8.8 & 3.3 & \\
\hline Optical density at $750 \mathrm{~nm}$ & 0.003 & 0.004 & & 0.002 \\
\hline Dry matter $\left(\mathrm{kg} \cdot \mathrm{m}^{-3}\right)$ & 16.8 & 14.8 & 6.8 & 16.0 \\
\hline $\mathrm{pH}$ & 8.4 & 8.2 & & 8.8 \\
\hline Conductivity $\left(\mathrm{mS} . \mathrm{cm}^{-1}\right)$ & 23.7 & & 22.2 & 20.9 \\
\hline $\mathrm{Na}^{+} \quad\left(10^{-3} \mathrm{~kg} \cdot \mathrm{m}^{-3}\right)$ & 6695 & & 6120 & 5832 \\
\hline $\mathrm{Ca}^{2+} \quad$ (id.) & & & n.a. & n.a. \\
\hline $\mathrm{Mg}^{2+} \quad$ (id.) & & 47.4 & 26.3 & 44.9 \\
\hline $\mathrm{K}^{+} \quad$ (id.) & 85 & 87.2 & 83.7 & 93.0 \\
\hline Total cations (id.) & 6820 & 5695 & 6230 & 5970 \\
\hline $\mathrm{Cl}^{-} \quad$ (id.) & 8607 & 6695 & 7604 & 7036 \\
\hline $\mathrm{NO}_{3}^{-} \quad$ (id.) & 552 & 882 & 1004 & 948 \\
\hline $\mathrm{SO}_{4}^{2-}$ & 50.5 & 30.3 & 911 & 135 \\
\hline $\mathrm{PO}_{4}^{3-}$ & 148 & 121 & 144 & 142 \\
\hline Total anions (id.) & 9357 & 7728 & 9663 & 8261 \\
\hline
\end{tabular}


Table 3. Experiments carried out and their operating conditions.

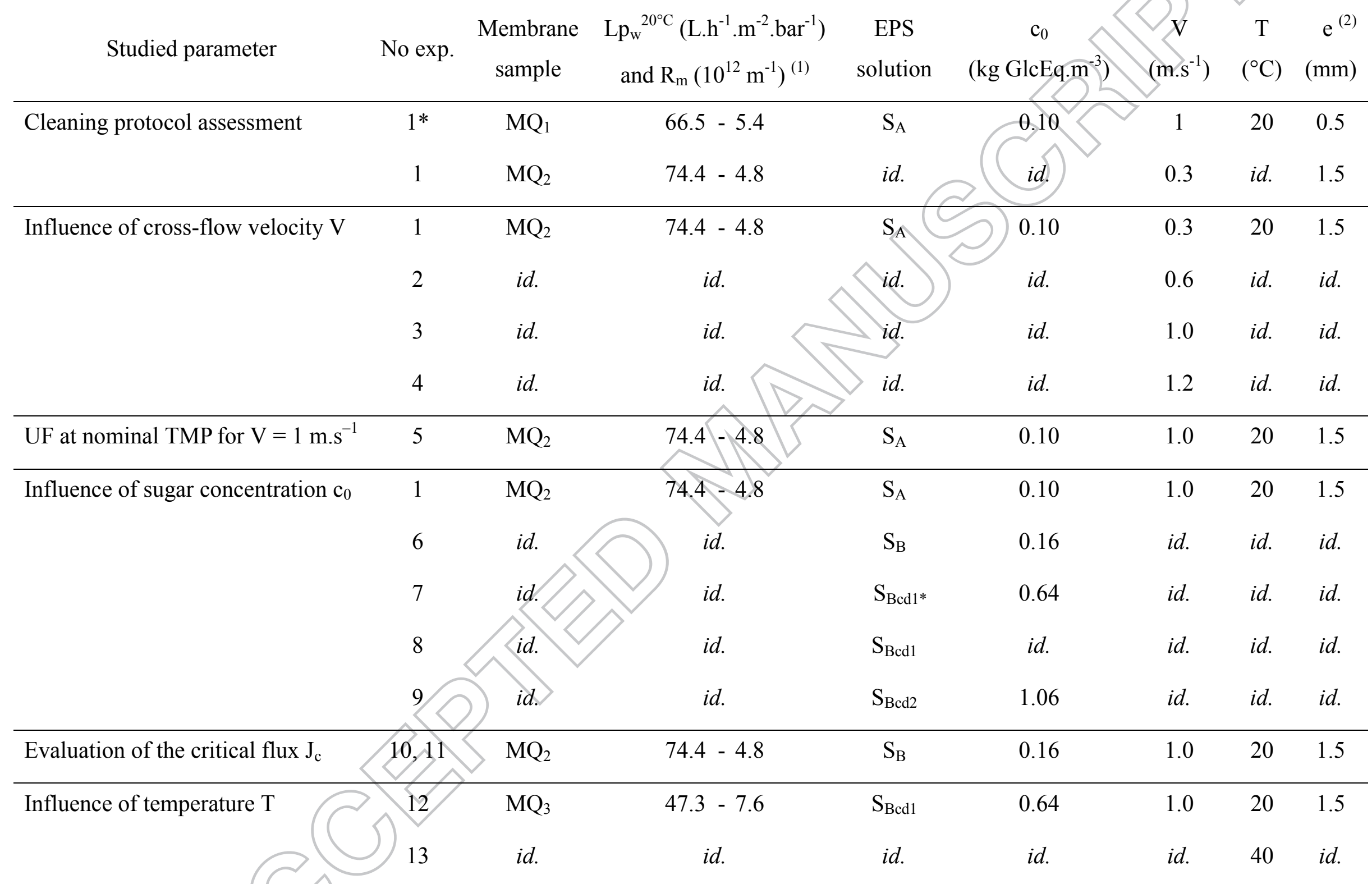

(1) Initial water permeability $\mathrm{Lp}_{\mathrm{w}}$ at $20^{\circ} \mathrm{C}$ and initial membrane resistance $\mathrm{R}_{\mathrm{m}}$

(2) Fluid vein of thickness e without $(\mathrm{e}=0.5 \mathrm{~mm})$ or with a feed spacer 46 mil $(\mathrm{e}=1.5 \mathrm{~mm})$. 
Table 4. Limiting permeate fluxes and TMP obtained during EPS fouling experiments at $20{ }^{\circ} \mathrm{C}$, with the membrane sample $\mathrm{MQ}_{2}$. Experimental conditions: exp. No. 1-4, e =1.5 mm with feed spacer, $\mathrm{VRR}=1$, EPS solution $\mathrm{S}_{\mathrm{A}}\left(\mathrm{c}_{0}=0.10 \mathrm{~kg} \mathrm{GlcEq} \cdot \mathrm{m}^{-3}\right), \mathrm{T}=20^{\circ} \mathrm{C}, \mathrm{V}=1.0 \mathrm{~m} \cdot \mathrm{s}^{-1}$.

\begin{tabular}{ccc}
\hline EPS solution & $\begin{array}{c}\text { EPS concentration } \\
\left(\mathrm{kg} \mathrm{GlcEq} \cdot \mathrm{m}^{-3}\right)\end{array}$ & $\begin{array}{c}\text { Limiting permeate flux } \\
\mathrm{J}_{\lim }\left(\mathrm{L}^{-} \mathrm{h}^{-1} \cdot \mathrm{m}^{-2}\right)\end{array}$ \\
\hline $\mathrm{S}_{\mathrm{A}}$ & 0.10 & 54.4 \\
$\mathrm{~S}_{\mathrm{B}}$ & 0.16 & 51.0 \\
$\mathrm{~S}_{\mathrm{Bcd} 1}$ & 0.64 & 38.0 \\
$\mathrm{~S}_{\mathrm{Bcd} 2}$ & 1.06 & 33.8
\end{tabular}


Table 5. Comparison of filtration performance of EPS solution and skim milk at close temperatures

\begin{tabular}{|c|c|c|c|c|c|}
\hline & $\begin{array}{c}\text { Membrane and } \\
\text { module }\end{array}$ & Run conditions ${ }^{(1)}$ & $\begin{array}{l}\text { Critical point } \\
\mathrm{TMP}_{\mathrm{c}} / \mathrm{J}_{\mathrm{c}}\end{array}$ & $\begin{array}{l}\text { Limiting point } \\
\qquad \mathrm{TMP}_{1} / \mathrm{J}_{1}\end{array}$ & 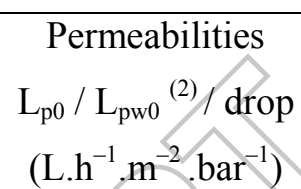 \\
\hline $\begin{array}{l}\text { EPS } \\
\text { solution } \\
\text { (this work) }\end{array}$ & $\begin{array}{c}\text { PES } 50 \mathrm{kDa} \\
\text { (Synder, mod. MQ) } \\
\text { Plate module } \\
\text { Rayflow } 100, \\
\text { fluid vein e }=1.5 \\
\text { mm with a } 46 \mathrm{mil} \\
\text { spacer }(1.168 \mathrm{~mm})\end{array}$ & $\begin{array}{c}\text { EPS: } 0.64 \mathrm{~kg} \mathrm{GlcEq} \cdot \mathrm{m}^{-3} \\
\text { Proteins: } 0.02 \mathrm{~kg} \cdot \mathrm{m}^{-3} \\
\text { Salts : } 15 \mathrm{~kg} \cdot \mathrm{m}^{-3} \\
\mathrm{~T}=40^{\circ} \mathrm{C} \\
\mathrm{V}=1.0 \mathrm{~m} \cdot \mathrm{s}^{-1} \\
\text { VRR }=1 \\
\text { Duration: } 50-80 \mathrm{~min}\end{array}$ & $\begin{array}{c}0.30 \mathrm{bar} \\
32 \mathrm{~L} \cdot \mathrm{h}^{-1} \cdot \mathrm{m}^{-2}\end{array}$ & , & \\
\hline $\begin{array}{l}\text { Skim milk } \\
\text { (Rabiller- } \\
\text { Baudry et } \\
\text { al. 2014) }\end{array}$ & $\begin{array}{c}\text { PES 5-10 kDa } \\
\text { (Koch, model } \\
\text { HFK-131) } \\
\text { Plate module } \\
\text { Rayflow 100, } \\
\text { fluid vein with a } 2 \\
\text { mm spacer }\end{array}$ & $\begin{array}{c}\text { Organic matter: } 32 \mathrm{~kg} \\
\text { prot.m }{ }^{-3}, 48 \mathrm{~kg} \text { lact.m }{ }^{-3} \\
\mathrm{~T}=46{ }^{\circ} \mathrm{C} \\
\mathrm{V}=0.3 \mathrm{~m} . \mathrm{s}^{-1} \\
\mathrm{VRR}=1 \\
\text { Duration: } 180 \mathrm{~min}\end{array}$ & $\mathrm{~m}^{-2}$ & $\begin{array}{c}3.5 \text { bar } \\
50 \mathrm{~L} \cdot \mathrm{h}^{-1} \cdot \mathrm{m}^{-2}\end{array}$ & $45 / 18 / 60 \%$ \\
\hline
\end{tabular}

(1) prot: proteins, lact: lactose, VRR: Volume Reduction Ratio, V: superficial velocity T: Temperature

${ }^{(2)} \mathrm{L}_{\mathrm{p} 0}$ : permeability at the lowest TMP; $\mathrm{L}_{\mathrm{pw} 0}$ : initial water permeability before filtration. 
Table A.2.1. Hydrodynamic conditions considered in spiral-wound, plate and frame and tubular modules; column a) real conditions in the module, column b) equivalent conditions considered in this work for the estimation of wall shear stress. Fluid properties assimilated to those of pure water at $20^{\circ} \mathrm{C}$ in all cases.

\begin{tabular}{|c|c|c|c|}
\hline Module & Real hydrodynamic conditions & $\begin{array}{l}\text { Equivalent hydrodynamic } \\
\text { conditions in free fluid vein }\end{array}$ & $\begin{array}{l}\tau_{\mathrm{w}}(\mathrm{Pa}) \\
\text { from (eq. A.2.2) }\end{array}$ \\
\hline Tubular (TUB) & $\begin{array}{l}\mathrm{V}=6.0 \mathrm{~m} \cdot \mathrm{s}^{-1} \\
\text { Cylindrical tube, } \mathrm{D}=12.7 \mathrm{~mm} \\
\text { (diameter of PCI membranes, } \\
\text { Filtration Group Corp., USA) }\end{array}$ & $\begin{array}{l}\text { Idem } \\
\left(\mathrm{D}_{\mathrm{h}}=12.710^{-3}\right.\end{array}$ & 86 \\
\hline Spiral-wound (SW) & $\begin{array}{l}\mathrm{V}=0.3 \mathrm{~m} \cdot \mathrm{s}^{-1} \\
\text { Spiral wound rectangular } \\
\text { channel, e }=1.5 \mathrm{~mm} \text { with a } 46 \\
\text { mil spacer }\end{array}$ & $\begin{array}{l}\mathrm{V}=1.0 \mathrm{~m} . \mathrm{s}^{-1} \\
\text { Flat rectangular free vein fluid, } \mathrm{e}= \\
0.5 \mathrm{~mm} \text {, without spacer }\left(\mathrm{D}_{\mathrm{h}}=10^{-3} \mathrm{~m}\right)\end{array}$ & 8 \\
\hline Plate and frame $(\mathrm{PF})$ & $\begin{array}{l}\mathrm{V}=1.0 \mathrm{~m} \cdot \mathrm{s}^{-1} \\
\text { Flat rectangular channel, } \mathrm{e}=1.5 \\
\mathrm{~mm} \text {, with a } 46 \text { mil spacer }\end{array}$ & $\begin{array}{l}\mathrm{V}(=1.0 / 0.3)=3.33 \mathrm{~m} \cdot \mathrm{s}^{-1} \\
\text { Flat free vein fluid, } \mathrm{e}=0.5 \mathrm{~mm} \text {, } \\
\text { without spacer }\left(\mathrm{D}_{\mathrm{h}}=10^{-3} \mathrm{~m}\right)\end{array}$ & 58 \\
\hline
\end{tabular}

